\title{
Inhomogeneities in non-hyperbolic one-dimensional invariant sets
}

\author{
by \\ Brian E. Raines (Oxford and Waco, TX)
}

\begin{abstract}
The topology of one-dimensional invariant sets (attractors) is of great interest. R. F. Williams [20] demonstrated that hyperbolic one-dimensional non-wandering sets can be represented as inverse limits of graphs with bonding maps that satisfy certain strong dynamical properties. These spaces have "homogeneous neighborhoods" in the sense that small open sets are homeomorphic to the product of a Cantor set and an arc. In this paper we examine inverse limits of graphs with more complicated bonding maps. This allows us to understand the topology of a wider class of spaces that includes both hyperbolic and non-hyperbolic attractors. Many of these spaces have the property that most small open sets are homeomorphic to the product of a Cantor set and an arc. The interesting "inhomogeneities" occur away from these neighborhoods. By examining the dynamics of the bonding maps that generate these spaces, we characterize the inhomogeneities, and we show that there is a natural nested hierarchy in the collection of these points that is topological.
\end{abstract}

1. Introduction. The topology of hyperbolic one-dimensional sets left invariant under a dynamical system is well understood. R. F. Williams [20] showed that these can be described as inverse limits of branched onemanifolds, and he developed much of the necessary theory for analyzing these spaces ([21], [22]). Anderson and Putnam [1] have shown that this class of spaces also includes the substitution tiling spaces. Recently, Barge and Diamond classified all such spaces that are orientable [3]. Topological invariants for the non-orientable case have also been found (see [23]).

However, very little is understood about the topology of one-dimensional invariant sets that are not hyperbolic. Perhaps the main difficulty in understanding the structure of these spaces is the fact that they tend to be quite complicated locally. Hyperbolic one-dimensional sets are solenoid-like in the sense that all sufficiently small neighborhoods are homeomorphic to the product of a Cantor set and an arc. Without the restriction that the space is hyperbolic, small neighborhoods can display very complicated structure.

2000 Mathematics Subject Classification: 37B45, 37E25, 54F15, 54H20. 
The points in these spaces that do not have such solenoid-like neighborhoods are the points of interesting topology.

In Section 2 we introduce new topological ideas to distinguish between points that have solenoid-like neighborhoods and those that do not in terms of linear covers. The utility of these definitions is demonstrated in Theorem 2.2 where we show that these definitions completely characterize the property of having a solenoid-like neighborhood.

In Sections 3 and 4 we apply Theorem 2.2 to give a sequence of theorems, Theorems 3.7, 4.1, and 4.6, that characterize points that have solenoid-like neighborhoods in terms of dynamics. Our characterization uses arbitrarily fine collections of linear covers called local chains. For spaces that can be modeled by an inverse limit of a graph and for sets left invariant under a dynamical system the structure of these covers is directly related to the dynamics of the bonding map or system that generates the space.

In Section 5, we demonstrate that the collection of points without solenoid-like neighborhoods can be partitioned into a nested ordinal hierarchy of sets, each level of which is preserved under homeomorphism. This leads to Theorem 5.3 and Corollary 5.4 which give both cardinal and order invariants for such spaces based only on the dynamics of the map that generated them. These theorems are generalizations of results of Barge and Martin [7] in which the dynamics of a unimodal interval map were shown to generate the endpoints of the inverse limit space giving a cardinal invariant for the associated inverse limit space. These spaces are good examples of non-hyperbolic attractors (see [6]).

Another well known family of non-hyperbolic attractors are the Hénon attractors. Most of the full attracting sets for maps in the Hénon family with dissipation parameter near zero appear to be locally solenoid-like. Barge and Holte [4] showed that many of the attractors for the Hénon family are inverse limits of unimodal maps of the interval. In [2], the authors remark that computer-aided investigation of the structure of the transitive Hénon attractors seems to indicate that these spaces are locally solenoidlike. However upon magnification many of these regions appear to have "hooks". They go on to demonstrate that, for a family of conceptual models for the Hénon attractors, inverse limits of tent maps, there is a dense $G_{\delta}$ set of parameters for which these spaces are nowhere locally solenoid-like. In fact they are "locally universal" in the sense that every neighborhood contains a homeomorph of every member of the family. On the other extreme, it is well known that there is a dense set of parameters for which the space is locally solenoid-like, except at its (finitely many) endpoints. These two cases are far from exhaustive, and in Sections 6 and 7 we will apply the results from previous sections to address the remaining case: that of spaces that have some neighborhoods homeomorphic to the product of a 
Cantor set and an arc but infinitely many points that have no such neighborhood.

Following Barge and Holte [4], in Sections 6 and 7 we focus on the structure of inverse limits of unimodal interval maps. In Section 6, we prove that given a locally eventually onto unimodal map $f$ with critical point $c$, and a point $x \in \lim \{[0,1], f\}, x$ has a neighborhood homeomorphic to a Cantor set of arcs if, and only if, $x_{n} \notin \omega_{f}(c)$ for infinitely many $n \in \mathbb{N}$. In Section 7, we end the paper with a collection of examples of unimodal maps, tent maps in fact, that illustrate some of the various topological structures possible in $\omega_{f}(c)$ and the resulting structure of the inhomogeneities in $\lim _{\longleftarrow}\{[0,1], f\}$.

2. Preliminary definitions and graph maps. We give a few definitions here, and introduce the others as needed. For standard definitions from topology or dynamics see [13] or [8]. If $f$ is a function, $U$ is a set, and $\mathcal{U}$ is a collection of sets, we will abuse notation throughout the paper by using $f(U)$ to mean the collection of points $\{f(x): x \in U\}$ and $f(\mathcal{U})=\{f(U): U \in \mathcal{U}\}$. We denote the closure of a set $A$ by $\bar{A}$. We call a compact, connected, metric space a continuum, and we call a compact connected subset of a continuum a subcontinuum.

Let $X$ be a topological space and $x \in X$. Let $V$ be an open set containing $x$. Call a finite collection $\mathcal{U}=\left\{U_{1}, \ldots, U_{n}\right\}$ of open subsets of $V$ a linear cover provided $\bar{U}_{i} \cap \bar{U}_{j} \neq \emptyset$ if, and only if, $|i-j|<2$. We will call the elements of such a linear cover links. If mesh $(\mathcal{U})<\varepsilon$ then we call $\mathcal{U}$ a linear $\varepsilon$-cover. Call a finite collection $\mathcal{U}=\left\{\mathcal{U}_{1}, \ldots, \mathcal{U}_{m}\right\}$ of linear covers of $V$, where $\mathcal{U}_{i}=\left\{U_{1}^{i}, \ldots, U_{p_{i}}^{i}\right\}$, a local chaining of $V$ if $\bigcup_{i \leq m} \mathcal{U}_{i}$ covers $V$ and $\overline{U_{j}^{i}} \cap \overline{U_{l}^{k}} \neq \emptyset$ if, and only if, $i=k$ and $|j-l|<2$. Call each element $\mathcal{U}_{i}$ of a local chaining $\mathcal{U}$ of $V$ a strand of $\mathcal{U}$. If each strand of $\mathcal{U}$ is a linear $\varepsilon$-cover, call $\mathcal{U}$ a local $\varepsilon$-chaining of $V$.

Let $\mathcal{C}=\left\{C_{1}, \ldots, C_{n}\right\}$ and $\mathcal{D}=\left\{D_{1}, \ldots, D_{m}\right\}$ be linear covers such that for each $i \leq m$ there is a $j \leq n$ such that $\bar{D}_{i} \subseteq C_{j}$. Then we say $\mathcal{D}$ refines $\mathcal{C}$ and we write $\mathcal{D} \leq \mathcal{C}$.

If $\mathcal{U}=\left\{\mathcal{U}_{1}, \ldots, \mathcal{U}_{n}\right\}$ and $\mathcal{V}=\left\{\mathcal{V}_{1}, \ldots, \mathcal{V}_{m}\right\}$ are local chainings of $V$ with the property that

(1) every strand of $\mathcal{V}$ refines exactly one strand of $\mathcal{U}$,

(2) every strand of $\mathcal{U}$ is refined by some strand of $\mathcal{V}$,

then we say $\mathcal{V}$ refines $\mathcal{U}$ and we write $\mathcal{V} \leq \mathcal{U}$. The mesh of a local chaining is the largest mesh of its strands.

$X$ is locally chainable at $x$ iff there is a neighborhood $U$ of $x$ and a sequence $\left\{\mathcal{C}_{i}\right\}_{i=1}^{\infty}$ of local chainings of $U$ such that 
(1) $\operatorname{mesh}\left(\mathcal{C}_{i}\right) \rightarrow 0$ as $i \rightarrow \infty$,

(2) $\mathcal{C}_{i} \leq \mathcal{C}_{i-1}$

We will say that the neighborhood $U$ is locally chainable in this case.

Let $\mathcal{U}$ be a local chaining of some $U \subseteq X$. Let $\mathcal{L}=\left\{L_{1}, \ldots, L_{p}\right\}$ be a linear cover that refines some strand $\mathcal{U}_{i}$ of $\mathcal{U}$. Let $L$ be a link of $\mathcal{U}_{i}$. We say that $\mathcal{L}$ turns in $L$ provided there is a $\operatorname{link} M$ in $\mathcal{U}_{i}$, adjacent to $L$, and integers $a$ and $b$ with $1 \leq a<b-1<b \leq p$ such that

(1) $L_{a}, L_{b} \subseteq M$

(2) $L_{j} \subseteq L-M$ for some $a<j<b$,

(3) $\bigcup_{i=a}^{b} L_{i} \subseteq L \cup M$.

We will then call $L$ a local turnlink, or just a turnlink. If every local $\varepsilon$ chaining of $U$ that refines $\mathcal{U}_{i}$ has a turnlink in $L$ then we call $L$ a local essential turnlink, or just an essential turnlink.

Let $\mathcal{C}$ be a linear cover in some space $X$ and let $\mathcal{D}$ be a linear cover that refines $\mathcal{C}$. Let $\mathcal{C}^{\prime}=\left\{C_{i}, C_{i+1}, \ldots, C_{i+k}\right\}$ be a subset of $\mathcal{C}$ and let $\mathcal{D}^{\prime}=$ $\left\{D_{j}, D_{j+1}, \ldots, D_{j+l}\right\}$ be a subset of $\mathcal{D}$ that refines $\mathcal{C}^{\prime}$ with $\bar{D}_{j} \subseteq C_{i}$. Suppose that we can find an integer $m_{1}$ such that

(1) $D_{j+m_{1}} \cap C_{i} \neq \emptyset$,

(2) $\bar{D}_{j+m_{1}} \subseteq C_{i+1}$

(3) $\bar{D}_{j+p} \subseteq C_{i}$ for all $0 \leq p<m_{1}$,

(4) $D_{j+q} \cap C_{i}=\emptyset$ for all $m_{1}<q \leq l$.

Inductively, given such an $m_{r-1}$ we can define $m_{r}$ larger than $m_{r-1}$ such that

(1) $D_{j+m_{r}} \cap C_{i+(r-1)} \neq \emptyset$,

(2) $\bar{D}_{j+m_{r}} \subseteq C_{i+r}$

(3) $\bar{D}_{j+p} \subseteq C_{i+(r-1)}$ for all $m_{r-1} \leq p<m_{r}$,

(4) $D_{j+q} \cap C_{i+(r-1)}=\emptyset$ for all $m_{r}<q \leq l$.

If $l \geq m_{k+1}$ then we say that $\mathcal{D}^{\prime}$ is straight in $\mathcal{C}^{\prime}$. Likewise if $D_{j} \subseteq C_{i+k}$ and we appropriately alter the above definition of the $m_{i}$ 's we still say that $\mathcal{D}^{\prime}$ is straight in $\mathcal{C}^{\prime}$.

Let $\mathcal{C}$ be a local chaining of a neighborhood $U$ of a point $x \in X$ of local chainability. Let $\mathcal{D}$ be another local chaining of $U$ such that $\mathcal{D} \leq \mathcal{C}$. If every strand of $\mathcal{D}$ is straight in every strand of $\mathcal{C}$ then we say that $\mathcal{D}$ is straight in $\mathcal{C}$.

Let $x$ be a point of local chainability of a metric space $X$ with neighborhood $U$. Call $x$ a local non-cut point of $X$ if for every neighborhood $V \subseteq U$ of $x$ and for every $\varepsilon>0$ there is a local $\varepsilon$-chaining $\mathcal{D}$ of $U$ that contains a strand $\mathcal{D}_{i}$ that has the closure of its first link contained inside $V$. Denote the set of local non-cut points of a space $X$ by $\operatorname{LNC}_{0}(X)$ or just by $\operatorname{LNC}(X)$. 
Let $X$ be a metric space with a point $x \in X$ such that $X$ is locally chainable at $x$. Let $U$ be a neighborhood of $x$ that is locally chainable. Call $x$ a folding point of $X$ if for every $\varepsilon>0$ there is a local $\varepsilon$-chaining $\mathcal{C}$ of $U$ that contains $x$ in an essential turnlink. Denote the set of folding points for a space $X$ by $\operatorname{Fold}_{0}(X)$ or just $\operatorname{Fold}(X)$.

We will show that the set of local non-cut points and of folding points contains all of the points $x \in X$ that are locally chainable and do not have solenoid-like neighborhoods. This will show that the inhomogeneities that can occur around points of local chainability are exactly folding points and non-cut points.

The next lemma describes explicitly the type of local chaining that occurs near a point that has a neighborhood that is solenoid-like. The proof is straightforward.

Lemma 2.1. Let $x$ be a point in a metric space $X$ such that $x$ has a neighborhood $U$ homeomorphic to the product of a Cantor set and an open arc, $(0,1)$. Then there exists a sequence $\left\{\mathcal{D}_{i}\right\}_{i=1}^{\infty}$ of local chainings of $U$ such that $\operatorname{mesh}\left(\mathcal{D}_{i}\right) \rightarrow 0$ as $i \rightarrow \infty$ and each $\mathcal{D}_{i}$ is labeled such that

(1) $\mathcal{D}_{1}=\left\{\mathcal{D}_{0}^{1}, \mathcal{D}_{1}^{1}\right\}$

(2) $\mathcal{D}_{i}=\left\{\mathcal{D}_{j}^{t_{1}, \ldots, t_{i-1}}: j \in\{0,1\}\right.$ and $t_{k} \in\{0,1\}$ for all $\left.k \leq i-1\right\}$,

(3) $\mathcal{D}_{j}^{t_{1}, \ldots, t_{i-1}}$ refines $\mathcal{D}_{t_{i-1}}^{t_{1}, \ldots, t_{i-2}}$ for all $t_{1}, \ldots, t_{i-1}, j \in\{0,1\}$,

(4) $\mathcal{D}_{j}^{t_{1}, \ldots, t_{i-1}}$ is straight in $\mathcal{D}_{t_{i-1}}^{t_{1}, \ldots, t_{i-2}}$ for all $t_{1}, \ldots, t_{i-1}, j \in\{0,1\}$. Moreover, $x$ is not in $\operatorname{LNC}(X)$.

Using the previous lemma we obtain the following result connecting the sets Fold $(X)$ and $\operatorname{LNC}(X)$ with the property of being solenoid-like.

Theorem 2.2. Let $X$ be a metric continuum. Then $x \in X$ has a neighborhood homeomorphic to the product of a zero-dimensional set and an arc if, and only if, $X$ is locally chainable at $x$ and $x \notin \operatorname{Fold}(X) \cup \operatorname{LNC}(X)$.

Proof. Suppose that $x$ has a neighborhood $U$ homeomorphic to the product of a Cantor set and an arc (the case that $U$ is homeomorphic to the product of another zero-dimensional set and an arc is easier so we do not handle it). Then clearly $X$ is locally chainable at $x$. Let $\left\{\mathcal{D}_{i}\right\}_{i=1}^{\infty}$ be the sequence of local refining chainings for $U$ guaranteed by Lemma 2.1. Suppose that $x \in \operatorname{Fold}(X)$. By Lemma 2.1, $x \notin \operatorname{LNC}(X)$, so let $\varepsilon>0$ and $\delta>0$ be small enough, with $\varepsilon>\delta$, so that no $\delta$-local chaining of $U$ has a strand with the closure of its first link in $B(x, \varepsilon) \subseteq U$. Let $\mathcal{C}$ be a local $\delta / 2$-chaining of $U$ such that $\mathcal{C}$ has a local essential turnlink $C$ containing $x$. Clearly $C \subseteq U$. Let $\gamma>0$ be such that every local $\gamma$-chaining of $X$ that refines $\mathcal{C}$ has a local turnlink in $C$. Let $n$ be large enough such that $\operatorname{mesh}\left(\mathcal{D}_{n}\right)<\gamma$. Then $\mathcal{D}_{n}$ contains a local turnlink in $C$. Let $\mathcal{D}_{i}^{n}$ be the strand of $\mathcal{D}_{n}$ containing $x$ in a 
turnlink $L$. Let $\mathcal{E}=\left\{E_{1}, \ldots, E_{m}\right\}$ be a linear cover that refines $\mathcal{D}_{i}^{n}$ such that $\mathcal{E}$ turns in $L \in \mathcal{D}_{i}^{n}$. Let $M$ be the link adjacent to $L$ given in the definition of local turnlink. Let $J$ be large enough such that $\operatorname{mesh}\left(\mathcal{D}_{J}\right)$ is less than the Lebesgue number for $\mathcal{E}$. Then some subset of a strand of $\mathcal{D}_{J}$ refines $\mathcal{E}$ and hence some strand of $\mathcal{D}_{J}$ turns in $\mathcal{D}_{n}$ (notice it cannot have its first or last link contained in $D_{i}^{n}$ so some "middle" link is in $D_{i}^{n}$ and since it refines $\mathcal{E}$ it must enter and exit $D_{i}^{n}$ via the same link, namely $\left.M\right)$, a contradiction. Hence $x \notin \operatorname{Fold}(X)$, so $x \notin \operatorname{Fold}(X) \cup \operatorname{LNC}(X)$.

Now suppose that $x \notin \operatorname{Fold}(X) \cup \operatorname{LNC}(X)$ and $X$ is locally chainable at $x$. Let $U$ be a neighborhood of $x$ with a sequence $\left\{\mathcal{D}_{i}\right\}_{i=1}^{\infty}$ of refining local chainings. We will show that each $\mathcal{D}_{i}$ is straight in $\mathcal{D}_{i-1}$. Then, since $X$ is a metric continuum, and since the intersection $K$ of the closures of all of the links of each strand is a compact connected subset of $X$ (i.e. a subcontinuum of $X$ ) it must be the case that $K$ is either an arc or a point, as these are the only continua which can be defined by a sequence of straight refining chains.

We will also show that there is another neighborhood $V$ of $x$ contained in $U$ such that each strand of $\mathcal{D}_{i}$ has its first and last link outside $V$ but several links with closures contained in $V$. This will demonstrate that each possible $K$ is in fact an arc. Then we will have shown that the intersection of these links in $V$ is the product of a zero-dimensional set and an arc. Hence $x$ will have a neighborhood (namely $V$ ) that is homeomorphic to the product of a zero-dimensional set and an arc.

Since $x$ is not in $\operatorname{LNC}(X)$, there is a neighborhood $V_{1} \subseteq U$ and a positive number $\delta$ such that no local $\delta$-chaining of $U$ has the closure of its first link in $V_{1}$. Since $x$ is not in $\operatorname{Fold}(X)$, let $\mathcal{D}_{i}$ be a local chaining of $U$ with some link $D$ containing $x$ so that its closure is contained inside $V_{1}$ and such that $D$ is not a local essential turnlink. Then for each $\varepsilon$ there is a local chaining $\mathcal{E}$ of $U$ that refines $\mathcal{D}_{i}$ such that $\mathcal{E}$ has no turnlinks inside $D$. Let $\mathcal{E}^{\prime}=\left\{E_{\alpha}, \ldots, E_{\alpha+\beta}\right\}$ be a subchain of $\mathcal{E}$ with $\bigcup_{k=0}^{\beta} E_{\alpha+k} \subseteq D$. Then there are no turnlinks in $\mathcal{E}^{\prime}$. Thus if $\mathcal{J}=\left\{J_{1}, \ldots, J_{c}\right\}$ is a refinement of $\mathcal{E}^{\prime}$ it must be the case that if $1 \leq \gamma_{1} \leq \gamma_{2} \leq c$ is defined so that (without loss of generality) $\bigcup_{k=\gamma_{1}}^{\gamma_{2}} J_{k} \subseteq E_{\alpha+r}$ for some $0 \leq r \leq \beta$ then there is no integer $d$ with $d<\gamma_{1}$ or $d>\gamma_{2}$ such that $J_{d} \subseteq E_{\alpha+r}$, because if there were such a value $d$ then the chain $\mathcal{J}$ would have turned in one of the links of $\mathcal{E}^{\prime}$. However none of the links of $\mathcal{E}$ are turnlinks. This implies that every refinement of such a local chaining is straight in the links of $\mathcal{E}$ that are contained in $D$.

Let $\mathcal{E}_{1}$ be a local chaining of $U$ that refines $\mathcal{D}_{i}$ and has no turnlinks in $D$. Let $E_{1}$ be a link of a strand of $\mathcal{E}_{1}$ that contains $x$. We will show that $E_{1}$ is the product of a zero-dimensional set and an arc. Let $\delta_{1}$ be the Lebesgue number for $\mathcal{E}_{1}$. Let $\mathcal{E}_{2}$ be a local chaining of $U$ with $\operatorname{mesh}\left(\mathcal{E}_{2}\right)<\delta_{1}$ and such that no link of $\mathcal{E}_{2}$ in $D$ is a turnlink (we can assume this because $D$ is not an 
essential turnlink). Then no strand of $\mathcal{E}_{2}$ turns in $E_{1}$ and since $E_{1} \subseteq V_{1}$, no strand of $\mathcal{E}_{2}$ starts (equivalently stops) in $E_{1}$. Hence every strand of $\mathcal{E}_{2}$ that meets $E_{1}$ runs straight through it (i.e. it either comes from a preceding link and leaves through the following link or vice versa). Let $\mathcal{F}_{2}=\left\{\mathcal{F}_{1}^{2}, \ldots, \mathcal{F}_{m_{2}}^{2}\right\}$ be the strands of $\mathcal{E}_{2}$ that pass through $E_{1}$.

We proceed inductively. Assume we have a local chaining $\mathcal{E}_{n}$ of $U$ defined such that no link of $\mathcal{E}_{n}$ in $D$ is a turnlink, each strand $\mathcal{F}_{i}^{n}$ of $\mathcal{E}_{n}$, for $i \leq m_{n}$, that meets $E_{1}$ is straight in the links of the strands $\mathcal{F}_{n-1}$ of $\mathcal{E}_{n-1}$ that meet $E_{1}$, and each such strand either originates in the link before $E_{1}$ and passes through to the link following it or vice versa. We can easily define $\mathcal{E}_{n+1}$ to have these properties. Clearly if we intersect the appropriate closures of links of "nested" strands in $E_{1}$ we will get an arc. Then the union of them is the product of some zero-dimensional set and an arc.

3. Local chainability of inverse limits of graph maps. In the previous section we gave a characterization of points in a space $X$ with a solenoid-like neighborhood in terms of being both locally chainable and not being in $\operatorname{Fold}(X)$ and $\operatorname{LNC}(X)$. Thus the interesting topology occurs precisely at the points $x$ such that either $X$ is not locally chainable at $x$ or $x \in \operatorname{Fold}(X)$ or $x \in \operatorname{LNC}(X)$.

We now leave the general setting of the previous section to consider the case where the space is an inverse limit of a graph. Let $f: G \rightarrow G$ be a continuous surjection that has finitely many turning points, $C=\left\{c_{1}, \ldots, c_{q}\right\}$. Let $V=\left\{v_{1}, \ldots, v_{r}\right\}$ be the set of branch points for $G$ and let $E=\left\{e_{1}, \ldots, e_{s}\right\}$ be the set of endpoints for $G$. Denote $\lim _{\longleftarrow}\{G, f\}$ by $X_{G}$.

In this section we use the dynamics of $f$ to examine the points of local chainability of $X_{G}$. It is quite easy to see that if $x$ is a branch point for $X_{G}$ then $X_{G}$ will not be locally chainable at $x$ and $x$ cannot have a solenoid-like neighborhood. Further if $x$ is a limit of branch points for $X_{G}$, then $X_{G}$ cannot be locally chainable at $x$. Unfortunately, this does not exhaust the possibilities. In 1972, W. T. Ingram [14] constructed a tree-like continuum $T_{\text {Ingram }}$ with no branch points that cannot be chained, i.e. $T_{\text {Ingram }}$ cannot be covered by a sequence of refining linear covers with vanishing mesh. If $x \in X_{G}$ has the property that $x$ is not a branch point but each neighborhood of $x$ contains arbitrarily small copies of $T_{\text {Ingram }}$ then $x$ is also not a point of local chainability for $X_{G}$. The problem of when $x$ is a point of local chainability of $X_{G}$ seems closely related to an old and hard problem in continuum theory known as the Span Zero Problem [19, Problem 8, Chapter 19]. We do not contribute to the solution of that problem here. But, interestingly, we demonstrate many conditions that when satisfied by the projections of a point $x \in X_{G}$ guarantee that $x$ is a point of local chainability. These conditions are all related to either projecting far away from branch points of $G$ 
or far away from recurrent branch points of $G$. This leads to the strongest theorem of the section regarding this property, Theorem 3.7, which implies, among other things, that even though $T_{\text {Ingram }}$ is not chainable, every point of $T_{\text {Ingram }}$ is a point of local chainability.

Occasionally we will assume that $f$ is "non-contracting" in the following sense:

if $B \subseteq G$ is connected and $A$ is some component of $f^{-1}(B)$ then $\operatorname{diam}(A) \leq \operatorname{diam}(B)$.

Let $\Gamma>0$ be small enough so that if $x, y \in C \cup V \cup E$ with $x \neq y$ then $d(x, y) \geq 2 \Gamma$. Call the union of $n$ arcs that share a single branch point in common an $n$-star or simply a star. Our choice of $\Gamma$ implies that every subcontinuum of $G$ with diameter less than $\Gamma$ must be either an arc or an $n$-star.

At times we will also assume that $f$ is eventually at least a two-pass map in the following sense:

( $\ddagger) \quad$ there is an integer $N$ so that for every subcontinuum $A$ of $G, f^{-n}(A)$ consists of at least two components for all $n \geq N$.

Both the assumptions $(\dagger)$ and $(\ddagger)$ are quite general - they ensure that we are considering maps with somewhat complicated dynamics that generate nontrivial inverse limit spaces. We will indicate in each result which assumption (if any) we are using.

We start with a few quite easy lemmas demonstrating neighborhoods that can be covered with local chains.

Lemma 3.1. Let $U$ be an arc in $G$ such that $f^{-n}(U)$ is a collection of disjoint arcs for each $n \in \mathbb{N}$. Then $\pi_{1}^{-1}(U)$ is locally chainable.

Proof. Let $U=U_{0}$ and let $U_{1}^{0}, \ldots, U_{n_{0}}^{0}$ be the components of $f^{-1}\left(U_{0}\right)$. There are finitely many such components because $f$ has finitely many turning points. Notice that each $U_{i}^{0}$ is an arc by assumption. Continuing, de-

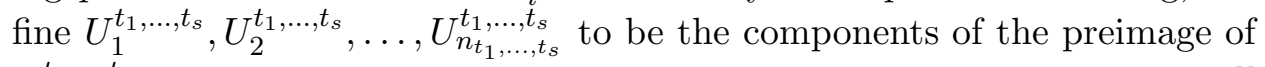
$U_{t_{s}}^{t_{1}, \ldots, t_{s-1}}$. Again these are finitely many and they are each arcs. Let $\Sigma \subseteq \mathbb{N}^{\mathbb{N}}$ be such that $\tau=\left(t_{1}, \ldots, t_{n}, \ldots\right) \in \Sigma$ if for each initial tuple $\left.\tau\right|_{q}=\left(t_{1}, \ldots, t_{q}\right)$ of $\tau$, there is an arc $U_{t_{q}}^{t_{1}, \ldots, t_{q-1}}$ defined as above.

There is a very natural way to define a chaining $\mathcal{C}_{0}$ of $U_{0}$, and given $\tau \in \Sigma$ and $q \in \mathbb{N}$ we can define a chaining $\mathcal{C}_{t_{1}, \ldots, t_{q}}$ to be a chaining of $U_{t_{q}}^{t_{1}, \ldots, t_{q-1}}$ such that $f\left(\mathcal{C}_{t_{1}, \ldots, t_{q}}\right)$ refines $\mathcal{C}_{t_{1}, \ldots, t_{q-1}}$. We can further impose upon this sequence of chains that $\operatorname{mesh}\left(\pi_{q}^{-1}\left(\mathcal{C}_{t_{1}, \ldots, t_{q}}\right)\right)<1 / 2^{q}$. Let $\mathcal{C}_{t_{1}, \ldots, t_{q}}^{\prime}=\pi_{q}^{-1}\left(\mathcal{C}_{t_{1}, \ldots, t_{q}}\right)$. Define $\mathcal{C}_{q}=\left\{\mathcal{C}_{\tau \mid q}^{\prime}: \tau \in \Sigma\right\}$. Then each $\mathcal{C}_{q}$ is a local chaining of $\pi_{1}^{-1}(U), \operatorname{mesh}\left(\mathcal{C}_{q}\right)<$ $1 / 2^{q}$, and $\mathcal{C}_{q}$ refines $\mathcal{C}_{q-1}$. Hence $\pi_{1}^{-1}(U)$ is a neighborhood that can be

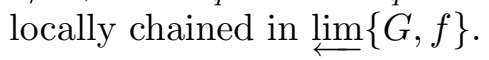


LEMMA 3.2. Let $U$ be a subcontinuum of $G$ such that $f^{-n}(U)$ is a collection of disjoint arcs for all $n \geq N$. Then $\pi_{N}^{-1}\left[f^{-N}(U)\right]$ is locally chainable.

Proof. Take each component of $f^{-N}(U)$ as the set $U_{0}$ in the proof given previously. This will lead to a local chaining of $\pi_{N}^{-1}\left[f^{-N}(U)\right]$.

The following theorem is a well known tool in the study of inverse limit spaces.

Theorem 3.3 ([15, Thm. 1.7], the Subsequence Theorem). Let $f_{i}: X \rightarrow$ $X$ be a mapping for every $i \in \mathbb{N}$. Then $\lim _{\longleftarrow}^{\lim }\left\{X, f_{i}\right\}$ is homeomorphic to $\lim _{\longleftarrow}\left\{X, f_{n_{i}}\right\}$ for any subsequence $\left\{n_{i}\right\}_{i \in \mathbb{N}}$ ( where by $f_{n_{i}}$ we mean the map $\left.\overleftarrow{f_{n_{i-1}}^{n_{i}}}=f_{n_{i-1}} \circ f_{n_{i-1}+1} \circ \cdots \circ f_{n_{i}-1}\right)$.

LEMMA 3.4. Let $U$ be a subcontinuum of $G$ such that for some increasing sequence $\left\{n_{i}\right\}_{i \in \mathbb{N}}$ of positive integers, $f^{-n_{i}}(U)$ does not meet $\left\{v_{1}, \ldots, v_{r}\right\}$. Then $\pi_{n_{1}}^{-1}\left[f^{-n_{1}}(U)\right]$ is locally chainable.

Proof. The proof is an easy application of the ideas found in the previous proofs and the Subsequence Theorem 3.3. Pull $U$ back to $f^{-n_{1}}(U)$. This is then a finite collection of arcs. So also is $f^{-n_{i}}(U)$ for each $i$. Hence $\pi_{n_{1}}^{-1}\left[f^{-n_{1}}(U)\right]$ is locally chainable in $\lim _{\longleftarrow}\left\{G, f^{n_{i}}\right\}$, which is homeomorphic to $\underset{\lim }{\longleftarrow}\{G, f\}$. The lemma follows.

LEMMA 3.5. Let $U$ be a subcontinuum of $G$ such that for infinitely many $N \in \mathbb{N}, U$ does not meet $\left\{f^{N}\left(v_{i}\right)\right\}_{i \leq r}$. If $M$ is the least such $N \in \mathbb{N}$, then $\pi_{M}^{-1}\left[f^{-M}(U)\right]$ is locally chainable.

Proof. The proof is an application of the previous lemma using $M$ as $n_{1}$ and defining each of the other $n_{i}$ 's appropriately.

TheOREM 3.6. Let $x \in X_{G}$ be such that $x_{n} \notin \omega(V)$ for all $n \in \mathbb{N}$. Then $X_{G}$ is locally chainable at $x$.

Proof. Follows immediately from the lemmas.

Theorem 3.7. (†) Let $x \in X_{G}$ be such that if $x_{n} \in \omega(V)$ then the set $V_{n}=\left\{v_{j_{1}}, \ldots, v_{j_{r}}: x_{n} \in \omega\left(v_{j_{i}}\right)\right\}$ has the property that $V_{n} \cap \omega(V)=\emptyset$. Then $X_{G}$ is locally chainable at $x$.

Proof. Since $\omega\left(v_{i}\right)$ is forward invariant, we can construct $V^{\prime}=\left\{v_{j_{1}}, \ldots\right.$ $\left.\ldots, v_{j_{r}}\right\} \subset V$ to be the collection of branch points such that $x_{n} \in \omega\left(v_{j_{i}}\right)$ for all $n$ larger than some fixed integer $L$. By hypothesis, none of the branch points in $V^{\prime}$ are in the $\omega$-limit set of a branch point. So for each $j_{i}$ there is a positive number $\gamma_{i}$ such that $B_{\gamma_{i}}\left(v_{j_{i}}\right) \cap \operatorname{orb}(V)=\left\{v_{j_{i}}\right\}$. Let $\gamma<\Gamma / 2$ be less than all the $\gamma_{i}$ 's and notice that if $U$ is a neighborhood of $x_{n}$ with $n \geq L$ and $\operatorname{diam}(U)<\gamma$ then, by assumption $(\dagger)$ about $f$, each component of an inverse image of $U$ must have diameter less than $\gamma$. So each component of an inverse image of $U$ meets $V^{\prime}$ at at most a single point. Moreover each 
component $A$ of a preimage of $U$ that contains a branch point $v_{j_{i}}$ meets $\operatorname{orb}\left(V^{\prime}\right)$ at that single branch point, and it has the property that $f^{-1}(A)$ is a disjoint collection of arcs.

Let $U$ be a $\gamma$-neighborhood around $x_{L}$. We will show that $\pi_{L}^{-1}(U)$ can be covered by a sequence of refining local chainings with vanishing mesh.

For each $v_{j_{i}} \in V^{\prime}$, let $\left\{n_{k}^{i}\right\}_{k \in \mathbb{N}}$ be defined so that $f^{n_{k}^{i}}\left(v_{j_{i}}\right) \rightarrow x_{L}$ as $k \rightarrow \infty$ and each $f^{n_{k}^{i}}\left(v_{j_{i}}\right)$ is in $U$. Let $M=\max \left\{n_{1}^{i}: i \leq r\right\}$. Then $\bigcup\left\{f^{-s}(U)\right.$ : $s \leq M\} \supseteq V^{\prime}$. Moreover, given any $v_{j_{i}} \in V^{\prime}$ and a star $S$ containing $v_{j_{i}}$, that is, a component of some $f^{-K}(U)$, we know:

(1) since $\operatorname{diam}(S)<\gamma, f^{-1}(S)$ is a disjoint collection of arcs,

(2) $f^{-(M+1)}(U)$ contains a collection of arcs that cover $f^{-1}(S)$ which is a subset of $f^{-(K+1)}(U)$,

(3) if $A$ is an arc in $f^{-1}(S)$ then $f^{-R}(A)$ is an arc for all $R>0$ due to our choice of $\gamma$.

This gives us a useful tool for constructing local chainings of $\pi_{L}^{-1}(U)$ because we know that the arcs in $f^{-(M+1)}(U)$ suffice to cover all of the possible inverse images of stars in $f^{-K}(U)$.

Let $\varepsilon_{1}>0$ be so small that if $\operatorname{diam}(V)<\varepsilon_{1}$ then $\operatorname{diam}\left(\pi_{L+M+1}^{-1}(V)\right)<$ $1 / 2$ and $\operatorname{diam}\left(\pi_{L+M+2}^{-1}(V)\right)<1 / 2$. Consider $f^{-(M+1)}(U)$. Clearly $f^{-(M+1)}(U)$ consists of a collection of disjoint stars $S_{1}^{1}, \ldots, S_{w_{1}}^{1}$, each centered at some branch point from $V^{\prime}$, and a disjoint collection of arcs $A_{1}^{1}, \ldots, A_{p_{1}}^{1}$. By our choice of $\gamma$ we know that $f^{-1}\left(S_{i}^{1}\right)$ is a disjoint collection of arcs and by our selection of $M$ we have $f^{-1}\left(S_{i}^{1}\right) \subseteq f^{-(M+1)}(U)$.

Let $\mathcal{E}_{i}^{1}$ be a linear cover of the arc $A_{i}^{1}$ with $\operatorname{mesh}\left(\mathcal{E}_{i}^{1}\right)<\varepsilon_{1}$ such that any $\mathcal{E}_{i}^{1}$ and $\mathcal{E}_{j}^{1}$ have links that have disjoint closures. Let

$$
\mathcal{C}_{i}^{1}=\pi_{L+M+1}^{-1}\left(\mathcal{E}_{i}^{1}\right)
$$

For each star $S_{k}^{1}$, let $A_{k_{1}}^{1}, \ldots, A_{k_{l}}^{1}$ be the subcollection of $A_{1}^{1}, \ldots, A_{p_{1}}^{1}$ that covers $f^{-1}\left(S_{k}^{1}\right)$. Then define

$$
\mathcal{D}_{k_{i}}^{1}=\pi_{M+L+2}^{-1}\left(E_{k_{i}}^{1}\right) \cap \pi_{M+L+1}^{-1}\left(S_{k}\right) .
$$

Let

$$
\mathcal{C}_{1}=\left\{\mathcal{C}_{i}^{1}\right\}_{i \leq p_{1}} \cup\left\{\mathcal{D}_{k_{i}}^{1}\right\}_{k \leq w_{1}, i \leq l} .
$$

We claim that $\mathcal{C}_{1}$ is a local chaining of $\pi_{L}^{-1}(U)$ with $\operatorname{mesh}\left(\mathcal{C}_{1}\right)<1 / 2$. Notice that $\mathcal{C}_{1}$ is a cover of $\pi_{L}^{-1}(U)$. To see this let $z \in \pi_{L}^{-1}(U)$. Then $\pi_{M+L+1}(z)$ is either in one of the arcs or in one of the stars. If it is in one of the arcs, say $A_{k}^{1}$, then $z$ is in a link of $\mathcal{C}_{k}^{1}$. If $z_{M+L+1}$ is in a star, say $S_{k}^{1}$, then $z_{M+L+2}$ is in an arc $A_{k_{d}}^{1}$, because of our original choice of $\gamma$. So $z$ is in a link of $\mathcal{D}_{k_{d}}^{1}$. Thus $\cup \mathcal{C}_{1}$ covers $\pi_{L}^{-1}(U)$. Clearly if $C_{s}^{1, i}$ is a link of $\mathcal{C}_{i}^{1}$ and $C_{t}^{1, h}$ is a link of $\mathcal{C}_{h}^{1}$ and $\overline{C_{s}^{1, i}}$ meets $\overline{C_{t}^{1, h}}$, then $h=i$ and $|s-t|<2$. The same is true for 
the strands $\mathcal{D}_{k_{s}}^{1}$. If there is a point $w$ in $\pi_{L}^{-1}(U)$ that is in the closure of a link of some $\mathcal{D}_{k_{s}}^{1}$ and in the closure of a link of some $\mathcal{C}_{t}^{1}$ then $w_{M+L+1}$ is contained in an arc $A_{t}^{1}$ and in a star $S_{k}^{1}$, a contradiction. Hence there is no such point $w$. Thus $\mathcal{C}_{1}$ is a local chaining for $\pi_{L}^{-1}(U)$.

Let $\varepsilon_{2}$ be small enough so that if $V$ is a subse of $G$ with $\operatorname{diam}(V)<\varepsilon_{2}$ then $\operatorname{diam}\left[\pi_{L+M+2}^{-1}(V)\right]<1 / 4$ and $\operatorname{diam}\left[\pi_{L+M+3}^{-1}(V)\right]<1 / 4$.

Consider $f^{-(M+2)}(U)$. It is a collection of $\operatorname{arcs} A_{1}^{2}, \ldots, A_{p_{2}}^{2}$ and stars $S_{1}^{2}, \ldots, S_{w_{2}}^{2}$. For each arc $A_{k}^{2}$ that is a preimage of an arc $A_{l}^{1}$, we can construct a linear cover $\mathcal{E}_{k}^{2}$ of $A_{k}^{2}$ with $\operatorname{mesh}\left(\mathcal{E}_{k}^{2}\right)<\varepsilon_{2}$ such that $f\left(\mathcal{E}_{k}^{2}\right)$ refines $\mathcal{E}_{l}^{1}$. For each arc $A_{m}^{2}$ that is the preimage of a star $S_{n}^{1}$, we have $A_{m}^{2}=A_{q}^{1}$ for some $q \leq p_{1}$. So we can refine $\mathcal{E}_{q}^{1}$ with a linear cover $\mathcal{E}_{m}^{2}$ of $A_{q}^{1}$ with $\operatorname{mesh}\left(\mathcal{E}_{m}^{2}\right)<\varepsilon_{2}$. This leads to the construction of a linear cover $\mathcal{E}_{i}^{2}$ with $\operatorname{mesh}\left(\mathcal{E}_{i}^{2}\right)<\varepsilon_{2}$ for each arc $A_{i}^{2}$ in $f^{-(M+2)}(U)$. Clearly if $f\left(\mathcal{E}_{k}^{2}\right)$ refines $\mathcal{E}_{l}^{1}$ then $\pi_{M+L+2}^{-1}\left(\mathcal{E}_{k}^{2}\right)$ refines $\mathcal{C}_{l}^{1}$. Also for the linear covers $\mathcal{E}_{m}^{2}$ that refine $\mathcal{E}_{q}^{1}$, $\pi_{M+L+2}^{-1}\left(\mathcal{E}_{m}^{2}\right)$ refines $\mathcal{D}_{q}^{2}$. So define

$$
\mathcal{C}_{s}^{2}=\pi_{M+L+2}^{-1}\left(\mathcal{E}_{s}^{2}\right)
$$

Notice also that each of the stars $S_{i}^{2}$ has the property that $f^{-1}\left(S_{k}^{2}\right)$ is a collection of $\operatorname{arcs} A_{k_{1}}^{2}, \ldots, A_{k_{l}}^{2}$. So define

$$
\mathcal{D}_{k_{r}}^{2}=\pi_{M+L+3}^{-1}\left(\mathcal{E}_{k_{r}}^{2}\right) \cap \pi_{M+L+2}^{-1}\left(S_{k}^{2}\right) .
$$

Again, let

$$
\mathcal{C}_{2}=\left\{\mathcal{C}_{i}^{2}\right\}_{i=1}^{p_{2}} \cup\left\{\mathcal{D}_{k_{i}}^{2}\right\}_{k \leq w_{2}, i \leq l} .
$$

By the same argument as given previously we find that $\mathcal{C}_{2}$ is a local chaining of $\pi_{L}^{-1}(U), \operatorname{mesh}\left(\mathcal{C}_{2}\right)<1 / 4$, and by construction, $\mathcal{C}_{2} \leq \mathcal{C}_{1}$.

It is not hard to see that we can inductively define chains $\mathcal{E}_{j}^{i}$ and construct refinements of them of sufficiently small mesh to build a local chaining $\mathcal{C}_{i+1}$ of $\pi_{L}^{-1}(U)$ such that $\operatorname{mesh}\left(\mathcal{C}_{i+1}\right)<1 / 2^{i+1}$ and $\mathcal{C}_{i+1} \leq \mathcal{C}_{i}$. Thus $X_{G}$ is locally chainable at $x$.

Corollary 3.8. Assume that $\omega(V) \cap V=\emptyset$. Then $X_{G}$ is locally chainable at every point $x$.

Each point $x \in X_{G}$ satisfies the assumptions of the previous theorem. Hence $X_{G}$ is locally chainable at $x$.

COROLlary 3.9. There are continua which are not chainable but which are locally chainable at every point.

Proof. Consider $T_{\text {Ingram }}$, the space described in [14]. The single branch point of the factor space is not recurrent, so the hypotheses of the previous theorem are satisfied for every point in $T_{\text {Ingram }}$. Hence every point of $T_{\text {Ingram }}$ is a point of local chainability. 
4. Inhomogeneities and dynamics. In this section we examine the other way in which a point cannot have a solenoid-like neighborhood: being a member of Fold $\left(X_{G}\right) \cup \operatorname{LNC}\left(X_{G}\right)$. We assume throughout that the points we are considering are points of local chainability, and we demonstrate that under assumption ( $\dagger$ ) the folding points for $X_{G}$ correspond exactly to the points that project infinitely often into $\omega(C)$, the $\omega$-limit set of the turning points for $f$, and the local non-cut points for $X_{G}$ correspond to the points that project infinitely often into $\omega(E)$, the $\omega$-limit set of the endpoints of $G$. If we add assumption $(\ddagger)$ then it follows that $\omega(E) \subseteq \omega(C)$ and so $\operatorname{LNC}\left(X_{G}\right) \subseteq \operatorname{Fold}\left(X_{G}\right)$. So, under assumptions $(\dagger)$ and $(\ddagger)$, we get the main result of the section: $x$ has a solenoid-like neighborhood if, and only if, $X_{G}$ is locally chainable at $x$ and $x_{n} \notin \omega(C)$ for all $n \in \mathbb{N}$.

We begin with an examination of points in $\operatorname{LNC}\left(X_{G}\right)$.

Theorem 4.1. (†) Let $x \in X_{G}$ be a point of local chainability. If for every $n \in \mathbb{N}$ there is an integer $i \leq s$ such that $x_{n} \in \omega\left(e_{i}\right)$ then $x \in \operatorname{LNC}\left(X_{G}\right)$.

Proof. Let $K \leq s$ be such that $x_{n} \in \omega\left(e_{K}\right)$ for all $n \in \mathbb{N}$. Let $U$ be an open set containing $x$ such that $U$ is covered by a sequence $\left\{\mathcal{C}_{i}\right\}_{i \in \mathbb{N}}$ of refining local chainings with vanishing mesh. If for every $x \in W \subseteq U$ there is one of these local chainings, say $\mathcal{C}_{i}$, such that the closure of the first (or equivalently last) link of a strand of $\mathcal{C}_{i}$ is contained in $W$ for infinitely many $i$ then clearly we are finished because then $x \in \operatorname{LNC}\left(X_{G}\right)$. So suppose that this is not the case. Let $x \in W \subseteq U$ be an open set such that each local chaining $\mathcal{C}_{i}$ with $i \geq Q$ from the sequence of refining local chainings of $U$ does not have the closure of any of its strands' first or last links in $W$.

Let $\varepsilon>0$ and let $N$ be large enough and $\delta<\Gamma / 4$, so that if $\mathcal{D}$ is a cover of $G$ with $\operatorname{mesh}(\mathcal{D})<\delta$ then $\operatorname{mesh}\left[\pi_{N}^{-1}(\mathcal{D})\right]<\min \{\operatorname{diam}(W) / 4, \varepsilon\}$. Let $\mathcal{D}$ be such a $\delta$-cover of $G$ and let $j$ be large enough so that $\operatorname{mesh}\left(\mathcal{C}_{j}\right)<\lambda$, the Lebesgue number for $\pi_{N}^{-1}(\mathcal{D})$. Let $M$ be large enough so that $\pi_{M}\left(\mathcal{C}_{j}\right)=\mathcal{C}_{j}^{\prime}$, with links $C_{s}^{j, u^{\prime}}=\pi_{M}\left(C_{s}^{j, u}\right)$, has the property that $\overline{C_{l}^{j, m^{\prime}}} \cap \overline{C_{p}^{j, n^{\prime}}} \neq \emptyset$ if, and only if, $m=n$ and $|l-p|<2$. Choose $m, p, p+1$ so that $x_{M} \in C_{p}^{j, m^{\prime}} \cup C_{p+1}^{j, m^{\prime}}$ and if $x_{M} \in \overline{C_{q}^{j, n^{\prime}}}$ then $n=m$ and $q \in\{p, p+1\}$. Thus there is a connected open set $W^{\prime}$ containing $x_{M}$ such that $W^{\prime} \subseteq C_{p}^{j, m^{\prime}} \cup C_{p+1}^{j, m^{\prime}}$ with $\operatorname{diam}\left(W^{\prime}\right)<\Gamma / 2$. Let $R$ be a positive integer large enough so that $f^{R}\left(e_{K}\right) \in W^{\prime}$. Notice that $f^{-R}\left(W^{\prime}\right)$ is a disjoint collection of $\operatorname{arcs} A_{1}, \ldots, A_{w}$ and stars $S_{1}, \ldots, S_{v}$ in $G$ such that each component of $f^{-R}\left(W^{\prime}\right)$ meets $V \cup E$ at at most one point, because each has diameter less than $\Gamma / 2$. So the component of $f^{-R}\left(W^{\prime}\right)$ that contains $e_{K}$ is an arc, since it cannot also contain a branch point for $G$. Call it $A_{r}$.

Consider the $(R+M)$ th projection of only the strand $\mathcal{C}_{m}^{j}$. Call it $\mathcal{C}_{m}^{j^{\prime \prime}}$. Then $C_{m}^{j^{\prime \prime}}$ is a linear cover of some subset $H$ of $G$. Let $B_{r}$ be the component 
of $H$ that contains $A_{r}$. Let $\mathcal{D}_{m}^{j}=\pi_{M+R}^{-1}\left[\mathcal{C}_{m}^{j^{\prime \prime}} \cap\left(H-B_{r}\right)\right]$. Then $\mathcal{D}_{m}^{j}$ is a linear cover of a subset of the portion of $U$ that is covered by the strand $\mathcal{C}_{m}^{j}$ of $\mathcal{C}_{j}$. Note that $\mathcal{D}_{m}^{j}$ only fails to cover points $z$ that have the property that $z_{R+M} \in B_{r}$. Define $\mathcal{E}$ to be a linear cover of $B_{r}$ such that $\mathcal{E} \leq \mathcal{C}_{m}^{j^{\prime \prime}}$ and:

(1) the first link of $\mathcal{E}$ contains $e_{K}$,

(2) the closures of the first three links of $\mathcal{E}$ are contained inside $A_{r}$ and miss $B_{r}-A_{r}$,

(3) the closures of the links of $\mathcal{E}$ are disjoint from the links of $\pi_{M+R}\left(\mathcal{C}_{s}^{j}\right)$, $s \neq m$ and $\pi_{M+R}\left(\mathcal{D}_{m}^{j}\right)$.

Let $\mathcal{E}^{\prime}=\pi_{M+R}^{-1}(\mathcal{E})$. Then $\left(\mathcal{C}_{j}-\left\{\mathcal{C}_{m}^{j}\right\}\right) \cup\left\{\mathcal{D}_{m}^{j}, \mathcal{E}^{\prime}\right\}$ is a local chaining of $U$ and the first link of $\mathcal{E}^{\prime}$ has its closure inside $W$. Hence $x \in \operatorname{LNC}\left(X_{G}\right)$.

Corollary 4.2. Let $x \in X_{G}$ be a point of local chainability. If for infinitely many $n \in \mathbb{N}$ there is an $i \leq s$ such that $x_{n} \in \omega\left(e_{i}\right)$ then $x \in \operatorname{LNC}\left(X_{G}\right)$.

Proof. $f(\omega(x))=\omega(x)$.

Under the "two-pass" assumption $(\ddagger)$, we have the following, which demonstrates that $\operatorname{LNC}\left(X_{G}\right)$ is just a special case of $\operatorname{Fold}\left(X_{G}\right)$.

Lemma 4.3. $(\dagger)(\ddagger)$ For each endpoint of $G, e_{i} \in \operatorname{orb}(C)$ so $\omega(E) \subseteq \omega(C)$.

Proof. Recall assumption ( $\ddagger)$. Let $N$ be large enough so that every subcontinuum $K$ of $G$ has at least two components in $f^{-N}(K)$. Assume $e_{i}$ is an endpoint so that no turning point $c_{j}$ is mapped to $e_{i}$. So $e_{i} \notin \operatorname{orb}(C)$. Consider the unique closed arc $A_{i}$ in $G$ containing $e_{i}$ of diameter $\Gamma / 4$. By the definition of $\Gamma, A_{i} \cap(E \cup V \cup C)=\left\{e_{i}\right\}$. If there is a point $z$ in $G$ that gets mapped to $e_{i}$ that is not an endpoint of $G$ then it must be a turning point for $f$. This does not occur because $e_{i} \notin \operatorname{orb}(C)$. So $f^{-1}\left(e_{i}\right)$ consists of only endpoints of $G$; moreover, $f^{-1}\left(e_{i}\right) \cap \operatorname{orb}(C)=\emptyset$. Thus $f^{-N}\left(e_{i}\right)$ is a collection of at least two endpoints for $G$. Let $X_{i} \subseteq\{1, \ldots, s\}$ be defined so that $k \in X_{i}$ if, and only if, $e_{k} \in f^{-N}\left(e_{i}\right)$. For $k \in X_{i}, e_{k} \notin \operatorname{orb}(C)$, so define $X_{i, k} \subseteq\{1, \ldots, s\}$ by $l \in X_{i, k}$ if, and only if, $e_{l} \in f^{-N}\left(e_{k}\right)$. If $k \neq m$ are two points of $X_{i}$ then $X_{i, k} \cap X_{i, m}=\emptyset$. Assuming we have defined $X_{i, t_{1}, \ldots, t_{n-1}}$, given a number $t_{n} \in X_{i, t_{1}, \ldots, t_{n-1}}$, let $X_{i, t_{1}, \ldots, t_{n-1}, t_{n}} \subseteq\{1, \ldots, s\}$ be defined so that $m \in X_{i, t_{1}, \ldots, t_{n-1}, t_{n}}$ if, and only if, $e_{m} \in f^{-N}\left(e_{t_{n}}\right)$. Thus we construct a sequence of sets that contain at least two integers with $X_{i, t_{1}, \ldots, t_{n-1}, t_{n}} \cap X_{i, t_{1}, \ldots, t_{n-1}, t_{n}^{\prime}}=\emptyset$ whenever $t_{n} \neq t_{n}^{\prime}$. Notice that for every $n$ there are at least $2^{n-1}$ disjoint subsets of $\{1, \ldots, s\}$ of size 2 . This contradicts the fact that we have only finitely many endpoints of $G$. Hence $e_{i} \in \operatorname{orb}(C)$.

Now we shift our attention to the points in $\operatorname{Fold}\left(X_{G}\right)$. 
TheORem 4.4. (†) Let $x \in X_{G}$ be a point of local chainability. If for each $n \in \mathbb{N}$ there is a positive integer $i$ such that $x_{n} \in \omega\left(c_{i}\right)$, then $x \in$ $\operatorname{Fold}\left(X_{G}\right)$.

Proof. Let $\left\{\mathcal{C}_{i}\right\}_{i=1}^{\infty}$ be a collection of local refining chainings of a neighborhood $U$ of $x$ such that $\operatorname{mesh}\left(\mathcal{C}_{i}\right) \rightarrow 0$ as $i \rightarrow \infty$. We will show that there is a natural number $N$ such that each $\mathcal{C}_{i}$ contains $x$ in a local essential turnlink for $i \geq N$. It will follow that $x \in \operatorname{Fold}\left(X_{G}\right)$.

Let $\mathcal{F}$ be an open cover of $G$ with $\operatorname{mesh}(\mathcal{F})<\Gamma / 4$. Let $\mathcal{F}^{\prime}=\pi_{1}^{-1}(\mathcal{F})$. This is an open cover of $X_{G}$. Let $\lambda_{0}$ be the Lebesgue number for this cover. Let $N$ be chosen large enough such that $\operatorname{mesh}\left(\mathcal{C}_{N}\right)<\lambda_{0}$.

Let $K$ be a natural number larger than $N$ and let $\mathcal{C}_{l}^{K}$ be the strand of $\mathcal{C}_{K}$ containing $x$ in a link. Let $r$ be such that if $x \in C_{j}^{K, l}$ then $j \in\{r, r+1\}$. Let $\lambda$ be less than the Lebesgue number for $\mathcal{C}_{K}$ such that any $\lambda$-refinement $\mathcal{E}$ of $\mathcal{C}_{K}$ must contain $x$ in a strand with the property that if $x \in E_{i}^{j}$ then $E_{u}^{j} \subseteq C_{r}^{K, l} \cup C_{r+1}^{K, l}$ for all $u=i-2, i-1, i, i+1, i+2$. Let $\mathcal{D}$ be some $\lambda$-refinement of $\mathcal{C}_{K}$. We will show that $\mathcal{D}$ contains a local turnlink in either $C_{r}^{K, l}$ or $C_{r+1}^{K, l}$.

Let $M$ be large enough such that $\pi_{M}(\mathcal{D})$ is a collection of disjoint linear covers of subsets of $G$ with non-adjacent links having disjoint closures. Since $\mathcal{D}$ refines $\mathcal{C}_{K}$, it also refines $\mathcal{C}_{N}$, so no component of a link of $\pi_{M}(\mathcal{D})$ contains two turning points for $f$. Let $\mathcal{D}_{T}$ be the strand of $\mathcal{D}$ containing $x$ in a link. Let $s$ be such that if $x \in D_{v}^{T}$ then $v \in\{s, s+1\}$. So $x_{M} \in \pi_{M}\left(D_{s}^{T}\right) \cup$ $\pi_{M}\left(D_{s+1}^{T}\right)=D_{s}^{T^{\prime}} \cup D_{s+1}^{T^{\prime}}$. Let $W$ be a connected open set containing $x_{M}$ such that $W \subseteq D_{s}^{T^{\prime}} \cup D_{s+1}^{T^{\prime}}$.

Since there is a turning point $c_{J}$ such that $x_{M} \in \omega\left(c_{J}\right)$, let $\left\{J_{1}, \ldots, J_{m}\right\}$ be the indices such that $x_{M} \in \omega\left(c_{J_{i}}\right)$ for all $i \leq m$. For each such $c_{J_{i}}$, there is an increasing sequence $\left\{n_{s}^{J_{i}}\right\}_{s=1}^{\infty}$ of integers such that $f^{n_{s}^{J_{i}}}\left(c_{J_{i}}\right) \rightarrow x_{M}$ as $s \rightarrow \infty$; additionally, we can pick these integers such that $f^{n_{s}^{J_{i}}}\left(c_{J_{i}}\right) \in W$ for each $s \in \mathbb{N}$. Let $w=n_{s}^{J_{i}}$ be the least such integer. Then $f^{w}\left(c_{J_{i}}\right) \in W$ and $f^{t}\left(c_{l}\right) \notin W$ for all turning points $c_{l}$, and for all $t<w$. Since $\operatorname{mesh}\left(\pi_{M}(\mathcal{D})\right)<$ $\Gamma / 2$, the diameter of $W$ can be made less than $\Gamma$. So $W$ does not meet more than one turning point for $f$, nor does any preimage of $W$.

Assume, without loss of generality, that $f^{w}\left(c_{J_{i}}\right) \in D_{s}^{T^{\prime}}$ and if $(a, b)$ is an arc containing $c_{J_{i}}$ that is not mapped completely into $D_{s}^{T^{\prime}}$ under $f^{w}$ then $f^{w}[(a, b)]$ meets $D_{s+1}^{T^{\prime}}$. Clearly if this is not the case we could have chosen a different index $s$ such that this was true. Since $f$ satisfies the noncontracting property $(\dagger)$, no component of a preimage of $W$ contains more than one turning point for $f$. Let $W^{\prime} \subseteq f^{-w}(W)$ be the component that contains $c_{J_{i}}$. Since $W^{\prime}$ contains no more than one turning point for $f$, there is an arc $(a, b)$ containing $c_{J_{i}}$ such that $f^{w}[(a, b)]$ is not contained in $D_{s}^{T^{\prime}}$ and 
even $f^{w}(a)=f^{w}(b)=z$. Then there must be components $A_{0}$ of $f^{-w}\left(D_{s}^{T^{\prime}}\right)$ and $A_{1}$ and $A_{2}$ of $f^{-w}\left(D_{s+1}^{T^{\prime}}\right)$ such that $c_{J_{i}} \in A_{0}, a \in A_{1}$ and $b \in A_{2}$. Let $\mathcal{E}=\left\{E_{1}, \ldots, E_{6}\right\}$ be a linear covering of $A_{0} \cup A_{1} \cup A_{2}$ with connected links such that $a \in E_{1} \subseteq A_{1}-A_{0}, c_{J_{i}} \in E_{3} \subseteq A_{0}$ and $b \in E_{6} \subseteq A_{3}-A_{0}$. Then $\pi_{M+w}^{-1}(\mathcal{E})=\mathcal{E}^{\prime}$ is a linear cover of a subset of $U$ that refines a strand of $\mathcal{D}$, namely $\mathcal{D}_{T}$, and $\mathcal{E}^{\prime}$ turns in $D_{s}^{T^{\prime}}$. Thus $D_{s}^{T^{\prime}}$ is a local turnlink and $\mathcal{C}_{K}$ contains $x$ in a local essential turnlink. Hence $x \in \operatorname{Fold}\left(X_{G}\right)$.

Corollary 4.5. (†) Let $x \in X_{G}$ be a point of local chainability. Suppose that for infinitely many $n \in \mathbb{N}$ there is an integer $i \leq q$ such that $x_{n} \in \omega\left(c_{i}\right)$. Then $x \in \operatorname{Fold}\left(X_{G}\right)$.

Proof. Let $m \in \mathbb{N}$. Choose $n \geq m$ and $i \leq q$ such that $x_{n} \in \omega\left(c_{i}\right)$. Then $x_{m}=f^{n-m}\left(x_{n}\right) \in f^{n-m}\left(\omega\left(c_{i}\right)\right)=\omega\left(c_{i}\right)$. Hence $x \in \operatorname{Fold}\left(X_{G}\right)$ by Theorem 4.4.

Theorem 4.6. (†) Let $x \in X_{G}$ be a point of local chainability. Suppose that for every $n \in \mathbb{N}$ there is no $i \leq q$ or $j \leq s$ such that $x_{n} \in \omega\left(c_{i}\right) \cup \omega\left(e_{j}\right)$. Then $x \notin \operatorname{Fold}\left(X_{G}\right) \cup \operatorname{LNC}\left(X_{G}\right)$.

Proof. Suppose $x \in X_{G}$ is a point of local chainability and for all $n \in \mathbb{N}$, $j \leq s$, and $i \leq q, x_{n} \notin \omega\left(c_{i}\right)$ and $x_{n} \notin \omega\left(e_{j}\right)$. Then for every $n$ there is an open set $U_{n}$ containing $x_{n}$ that is disjoint from $\left\{f^{m}\left(c_{j}\right)\right\}_{j \leq q, m \in \mathbb{N}} \cup$ $\left\{f^{m}\left(e_{j}\right)\right\}_{m \in \mathbb{N}, j \leq s}$. Let $U$ be a neighborhood of $x$ with a sequence $\left\{\mathcal{C}_{i}\right\}_{i \in \mathbb{N}}$ of local refining chains covering $U$ with $\operatorname{mesh}\left(\mathcal{C}_{i}\right) \rightarrow 0$ as $i \rightarrow \infty$. Notice that $\pi_{1}^{-1}\left(U_{1}\right) \cap U=U^{\prime}$ is also a neighborhood of $x$ and $\left\{\mathcal{C}_{i}^{\prime}\right\}_{i \in \mathbb{N}}$ can be constructed from $\left\{\mathcal{C}_{i}\right\}_{i \in \mathbb{N}}$ to be a sequence of local refining chains of $U^{\prime}$ with vanishing mesh.

Suppose that the theorem is false and that $x$ is in an essential turnlink of $\mathcal{C}_{1}^{\prime}$. Let $\gamma$ be small enough so that any local $\gamma$-refinement of $\mathcal{C}_{1}^{\prime}$ has a local turnlink in some link $C_{m}^{1, i}$ of $\mathcal{C}_{1}^{\prime}$ that contains $x$, and suppose $\gamma$ is less than the Lebesgue number for $\mathcal{C}_{1}^{\prime}$. Let $N$ be large enough so that $\pi_{N}\left[\mathcal{C}_{1}^{\prime}\right]$ is a collection of (not necessarily open) linear covers of a subset of $G$. Let $\delta$ be small enough so that any $\delta$-cover $\mathcal{E}$ of $G$ has $\operatorname{mesh}\left(\pi_{N}^{-1}(\mathcal{E})\right)<\gamma$. Let $\mathcal{D}$ be a collection of linear covers with connected links of the portion of $G$ covered by $\pi_{N}\left(\mathcal{C}_{1}^{\prime}\right)$. Then $\pi_{N}^{-1}(\mathcal{D})=\mathcal{D}^{\prime}$ has a local turnlink in $C_{m}^{1, i}$. Let $D_{s}^{K^{\prime}}$ and $D_{s+1}^{K^{\prime}}$ be links of $\mathcal{D}_{K}^{\prime}$, a strand of $\mathcal{D}^{\prime}$, contained in $C_{m}^{1, i}$ such that $D_{s}^{K^{\prime}}$ is a turnlink and some refinement turns in $D_{s}^{K^{\prime}}$ and enters and exits $D_{s}^{K^{\prime}}$ via $D_{s+1}^{K^{\prime}}$.

We will demonstrate that this cannot happen by refining $\mathcal{D}$ with a sequence of refining local chainings with vanishing mesh that do not turn in $D_{s}^{K^{\prime}}$, entering and exiting via $D_{s+1}^{K^{\prime}}$. This will imply that no refinement of $\mathcal{D}$ could turn in $D_{s}^{K^{\prime}}$ entering and exiting via $D_{s+1}^{K^{\prime}}$. 
The construction of such a sequence of refining local chains follows from the observation that since $U_{1}$ contains no points in $\left\{f^{m}\left(c_{j}\right)\right\}_{m \in \mathbb{N}, j \leq q} \cup$ $\left\{f^{m}\left(e_{j}\right)\right\}_{m \in \mathbb{N}, j \leq s}$, no inverse image of $U_{1}$ meets this set. So we have $D_{s}^{K}$ preceded by $D_{s-1}^{K}$ and followed by $D_{s+1}^{K}$. This will be true for every component of $f^{-n}\left(D_{s}^{K}\right)$ and $f^{-n}\left(D_{s-1}^{k}\right)$ and $f^{-n}\left(D_{s+1}^{K}\right)$. Since none of these components contain a turning point for $f$, there is no arc with endpoints being mapped under some $f^{m}$ into $D_{s+1}^{K}$ and an interior point being mapped under $f^{m}$ into $D_{s}^{K}$. Thus we can easily construct such a sequence of refining chains with vanishing mesh; call it $\left\{\mathcal{F}_{i}\right\}_{i \in \mathbb{N}}$. Hence $\mathcal{D}^{\prime}$ does not contain a turnlink in $C_{m}^{1, i}$, and $C_{m}^{1, i}$ is not an essential turnlink.

If $x \in \operatorname{Fold}\left(X_{G}\right)$ then for every $\varepsilon$ there is a local $\varepsilon$-chaining of $U_{1}$ containing $x$ in an essential turnlink. This would clearly imply that some link of $\mathcal{C}_{1}$ containing $x$ is an essential turnlink, which we showed was false. Hence $x \notin \operatorname{Fold}\left(X_{G}\right)$.

Moreover, suppose that $x \in \operatorname{LNC}\left(X_{G}\right)$. Then for every neighborhood $W \subseteq U$ of $x$ and for all $\varepsilon>0$ there is a local $\varepsilon$-chaining of $U$ with some strand that has the closure of its first link in $W$. Notice that $D_{s}^{K^{\prime}}$ is a neighborhood of $x$ and is contained in $U$. Suppose that $\mathcal{E}$ is a local chaining of $U$ with $\operatorname{mesh}(\mathcal{E})$ less than the Lebesgue number for $\mathcal{D}$. Also suppose that some strand $\mathcal{E}_{i}$ of $\mathcal{E}$ has the closure of its first link in $D_{s}^{K^{\prime}}$. Let $J$ be large enough so that $\mathcal{F}_{J} \leq \mathcal{E}$. Then some strand $\mathcal{F}_{t}^{J}$ of $\mathcal{F}_{J}$ refines $\mathcal{E}_{i}$. But no strand of $\mathcal{F}_{J}$ starts in $D_{s}^{K^{\prime}}$, so there is some link $F_{u}^{J, t}$ of $\mathcal{F}_{t}^{J}$ with $u \notin\left\{1,\left|\mathcal{F}_{t}^{J}\right|\right\}$ that is contained in $E_{1}^{i} \subseteq D_{s}^{K^{\prime}}$. Let $w$ be the least integer such that $E_{w}^{i} \cap D_{s}^{K^{\prime}}=\emptyset$. Then clearly there are integers $a, b$ with $a<u<b$ such that $F_{a}^{J, t}$ and $F_{b}^{J, t}$ are contained in $E_{w}^{i}$ and $F_{v}^{J, t} \subseteq E_{1}^{i}$ for all $a<v<b$. Thus $\mathcal{F}_{J}$ turns in $D_{s}^{K^{\prime}}$, a contradiction. Hence $x \notin \operatorname{LNC}\left(X_{G}\right)$.

Notice that if we had added assumption $(\ddagger)$ to the previous theorem we could have omitted the assumption that $x_{n} \notin \omega\left(e_{j}\right)$ for all $j \leq s$.

Corollary 4.7. $(\dagger)(\ddagger) \operatorname{LNC}\left(X_{G}\right) \subseteq \operatorname{Fold}\left(X_{G}\right) \subseteq \bigcap_{i \in \mathbb{N}} \pi_{i}^{-1}[\omega(C)]$.
Moreover if $\omega(V) \cap V=\emptyset$ then $\operatorname{Fold}\left(X_{G}\right)=\bigcap_{i \in \mathbb{N}} \pi_{i}^{-1}[\omega(C)]$.

Proof. This follows immediately from the previous two theorems.

Notice that the only points that might project always into $\omega(C)$ and not be in $\operatorname{Fold}\left(X_{G}\right)$ are those points where $X_{G}$ fails to be locally chainable.

Corollary 4.8. Let $x \in X_{G}$.

(1) $(\dagger) x$ has a neighborhood homeomorphic to the product of a zerodimensional set and an arc if, and only if, $X_{G}$ is locally chainable at $x$ and $x_{n} \notin \omega(C) \cup \omega(E)$ for some $n \in \mathbb{N}$. 
(2) $(\dagger)$ Suppose that $\omega(V) \cap V=\emptyset$. Then $x$ has a neighborhood homeomorphic to the product of a zero-dimensional set and an arc if, and only if, $x_{n} \notin \omega(C) \cup \omega(E)$ for some $n \in \mathbb{N}$.

(3) $(\dagger)(\ddagger) x$ has a neighborhood homeomorphic to the product of a zerodimensional set and an arc if, and only if, $X_{G}$ is locally chainable at $x$ and $x_{n} \notin \omega(C)$ for some $n \in \mathbb{N}$.

(4) $(\dagger)(\ddagger)$ Suppose that $\omega(V) \cap V=\emptyset$. Then $x$ has a neighborhood homeomorphic to the product of a zero-dimensional set and an arc if, and only if , $x_{n} \notin \omega(C)$ for some $n \in \mathbb{N}$.

Proof. The corollary follows from Theorem 2.2 combined with the characterization of $\operatorname{Fold}\left(X_{G}\right)$ and $\operatorname{LNC}\left(X_{G}\right)$ given in the previous corollary.

5. The nested hierarchy of inhomogeneities. We now shift our attention to the structure of $\operatorname{Fold}\left(X_{G}\right)$ and $\operatorname{LNC}\left(X_{G}\right)$. For a set $A$ denote the limit points of $A$ by $A^{\prime}$. Denote $\omega(A)$ by $\omega^{0}(A)$. For each non-limit ordinal $\alpha$, define $\omega^{\alpha}(A)=\omega^{\alpha-1}(A)^{\prime}$, and for $\lambda$ a limit ordinal let $\omega^{\lambda}(A)=\bigcap_{\beta<\lambda} \omega^{\beta}(A)$.

We can extend this idea to both $\operatorname{LNC}(X)$ and $\operatorname{Fold}(X)$. Inductively define $\mathrm{LNC}^{\alpha}(X)$ to be the collection of limit points of $\mathrm{LNC}^{\alpha-1}(X)$ for each nonlimit ordinal $\alpha$, and for $\lambda$ a limit ordinal let $\operatorname{LNC}^{\lambda}(X)=\bigcap_{\alpha<\lambda} \operatorname{LNC}^{\alpha}(X)$. Similarly, we inductively define Fold ${ }^{\alpha}(X)$ to be the collection of limit points of $\operatorname{Fold}^{\alpha-1}(X)$ for each non-limit ordinal $\alpha$, and for $\lambda$ a limit ordinal let $\operatorname{Fold}^{\lambda}(X)=\bigcap_{\alpha<\lambda} \operatorname{Fold}^{\alpha}(X)$. If $x \in \operatorname{Fold}^{\alpha}(X)$ but $x \notin \operatorname{Fold}^{\beta}(X)$ for all $\beta>\alpha$ then we call $x$ an $\alpha$-folding point.

This is simply the definition of the iterated Cantor-Bendixson derivatives of each of these sets (see [16]). For any Polish space, $X$, there must exist a countable ordinal $\alpha_{0}$ such that $X^{\alpha_{0}}=X^{\alpha}$ for all $\alpha>\alpha_{0}$ [16, Theorem 6.11]. This $\alpha_{0}$ is called the Cantor-Bendixson rank of $X$ and it is denoted by $|X|_{\mathrm{CB}}$.

The following two lemmas are immediate consequences of the definitions.

Lemma 5.1. Let $X$ be a metric space. Then $\operatorname{Fold}^{\alpha}(X) \supseteq \operatorname{Fold}^{\beta}(X)$ and $\operatorname{LNC}^{\alpha}(X) \supseteq \operatorname{LNC}^{\beta}(X)$ for all ordinals $\alpha<\beta$.

Lemma 5.2. Let $X$ and $Y$ be metric spaces and $h: X \rightarrow Y$ a homeomorphism. Then $h\left[\operatorname{Fold}^{\alpha}(X)\right]=\operatorname{Fold}^{\alpha}(Y)$ and $h\left[\operatorname{LNC}^{\alpha}(X)\right]=\operatorname{LNC}^{\alpha}(Y)$.

Combining this lemma with Corollary 4.8 we see that the points of local chainability that fail to have solenoid-like neighborhoods occur in a nested ordinal hierarchy of sets, each level of which is preserved under homeomorphism. This significantly strengthens previous results regarding invariants for inverse limits of graphs. What is more, we also have an order invariant for these spaces. If $X_{G}$ has the property that $\left|\operatorname{Fold}\left(X_{G}\right)\right|_{\mathrm{CB}}=\gamma$ then every homeomorphic space has the same property. The same result is true for 
$\operatorname{LNC}\left(X_{G}\right)$. We summarize these comments in the following theorem. By $|A|$ we simply mean the cardinality of $A$.

TheOREm 5.3. (†) Let $f: G \rightarrow G$ be a continuous surjection of a finite graph $G$ satisfying $(\dagger)$ with finite sets $C$ of turning points and $E$ of endpoints. Let $\alpha$ be an ordinal, and let

$$
\beta_{F}=\left|\operatorname{Fold}\left(\varliminf_{\longleftarrow}^{\lim }\{G, f\}\right)\right|_{\mathrm{CB}}, \quad \beta_{L}=\left|\mathrm{LNC}\left(\varliminf_{\longleftarrow}^{\lim }\{G, f\}\right)\right|_{\mathrm{CB}} .
$$

Then the following express topological properties for $\lim _{\longleftarrow}\{G, f\}$ :
(1) $\beta_{F}$;
(3) $\mid$ Fold $^{\alpha}\left(\lim _{\longleftarrow}\{G, f\}\right) \mid$;
(2) $\beta_{L}$;
(4) $\left|\operatorname{LNC}^{\alpha}\left(\lim _{\longleftarrow}\{G, f\}\right)\right|$.

For many maps of graphs it can be shown that $\left|\operatorname{Fold}^{\alpha}\left(\lim _{\longleftarrow}\{G, f\}\right)\right|=$ $\left|\omega^{\alpha}(C)\right|$ and $\left|\operatorname{LNC}^{\alpha}\left(\varliminf_{\longleftarrow}^{\lim }\{G, f\}\right)\right|=\left|\omega^{\alpha}(E)\right|$. We call such maps $f$ wellfounded and we give a family of examples of well-founded interval maps in Section 7.

Corollary 5.4. (†) Let $f: G \rightarrow G$ be a continuous surjection of $G$ with finitely many turning points satisfying assumption $(\dagger)$ such that $\omega(V) \cap$ $V=\emptyset$ and suppose $f$ is well-founded. Let $C, E, \alpha, \beta_{F}$ and $\beta_{L}$ be as in Theorem 5.3. Then the following express topological properties of $\varliminf_{\longleftarrow}\{G, f\}$ :
(1) $\beta_{F}$;
(3) $\left|\omega^{\alpha}(C)\right|$;
(2) $\beta_{L}$;
(4) $\left|\omega^{\alpha}(E)\right|$.

Proof. If $\omega(V) \cap V=\emptyset$, then, by Corollary 3.8, every point of the inverse limit $\lim _{\longleftarrow}\{G, f\}$ is a point of local chainability. Thus by Theorem 2.2 the only points in $\lim \{G, f\}$ that do not have solenoid-like neighborhoods

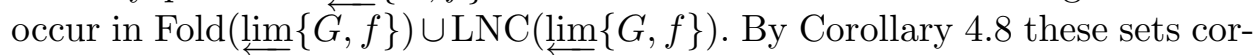
respond directly to $\omega(C)$ and $\omega(E)$. Since $f$ is well-founded, for any ordinal $\alpha$, Fold $^{\alpha}\left(\lim _{\lfloor}\{G, f\}\right)$ and $\operatorname{LNC}^{\alpha}\left(\varliminf_{\longleftarrow}\{G, f\}\right)$ correspond to $\omega^{\alpha}(C)$ and $\omega^{\alpha}(E)$, respectively. The result then follows by Lemma 5.2.

In Section 7 we give examples of well-founded interval maps $f$ such that $\underset{\longleftarrow}{\longleftarrow}\{[0,1], f\}$ exhibits such cardinal and order invariants.

6. Maps of the interval. A space $X$ is chainable if $X$ can be covered by a sequence of refining linear covers with vanishing mesh. Each such linear cover is called a chaining of $X$ or just a chain. The elements of a chain are called its links. The largest diameter of a chain's link is called its mesh. If $\operatorname{mesh}(\mathcal{C})<\varepsilon$, for a chain $\mathcal{C}$, then we call $\mathcal{C}$ an $\varepsilon$-chain.

The following is a well known theorem.

Theorem $6.1([15])$. Let $f: X \rightarrow X$ be a continuous mapping on a chainable continuum $X$. Then $\lim _{\longleftarrow}\{X, f\}$ is chainable. 
Notice that if $X$ is chainable and $x \in X$ then we can cover any neighborhood $U$ of $x$ with a sequence of local chainings with vanishing mesh. Thus $X$ is locally chainable at every point. It takes very little modification for all of the results from the previous sections to be applied to chainable continua. The main modification is that we no longer need to concern ourselves about the local chainability at a point.

If $X$ is a chainable continuum then $x$ is an endpoint of $X$ provided that for every $\varepsilon>0$ there is a chaining of $X$ with mesh less than $\varepsilon$ containing $x$ in its first link.

Lemma 6.2. Let $X$ be chainable. Then $x \in \operatorname{LNC}(X)$ if , and only if, $x$ is an endpoint of $X$.

Proof. If $x \in \operatorname{LNC}(X)$ then by the definition, for all $\varepsilon>0$ there is a chaining of $X$ with its first link containing $x$. Thus $x$ is an endpoint for $X$. The converse is similarly straightforward.

Let $\operatorname{End}(X)$ denote the set of endpoints for $X$. Combining the previous lemma with Theorem 2.2 we immediately have the following result.

Theorem 6.3. Let $X$ be a chainable continuum. Then $x \in X$ has a neighborhood homeomorphic to the product of a zero-dimensional set and an arc if , and only if,$x \notin \operatorname{End}(X) \cup \operatorname{Fold}(X)$.

If we assume $f:[0,1] \rightarrow[0,1]$ is a continuous function with a finite set $C$ of turning points, then $\lim _{\longleftarrow}\{[0,1], f\}$ is a chainable continuum so we can combine the previous theorem with Corollary 4.8 to obtain the following theorem.

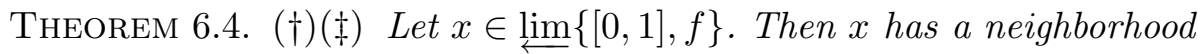
homeomorphic to the product of a zero-dimensional set and an arc if, and only if, $x_{n} \notin \omega(C)$ for some $n \in \mathbb{N}$.

Using the same definitions found in Section 5 and the previous results we have the following theorem.

Theorem 6.5. ( $\dagger$ ) Let $f$ be well-founded. Let $\alpha$ be an ordinal and let $\beta_{C}=|\omega(C)|_{\mathrm{CB}}$. Then the following express topological properties of the inverse limit $\lim _{\longleftarrow}\{[0,1], f\}$ :

(1) $\beta_{C}$;

(2) $\left|\omega^{\alpha}(C)\right|$.

A continuum $X$ is indecomposable provided that if $X=A \cup B$ where $A$ and $B$ are subcontinua then one of $A$ or $B$ is $X$.

Lemma 6.6. Let $K$ be an indecomposable chainable continuum and $x$ an endpoint of $X$. Then $x$ is a folding point. 
Proof. Let $\mathcal{C}$ be a chaining of $K$ with links $L$ and $M$ such that $x \in L$ and $x \notin M$ for all $M \in \mathcal{C} \backslash\{L\}$. Let $\gamma>0$ be less than the Lebesgue number for $\mathcal{C}$ such that any $\gamma$-chaining of $K$ has at least two links. Let $\mathcal{D}$ be such a $\gamma$-chaining of $K$. We will show that $\mathcal{D}$ has a turnlink in $L$.

Let $\mathcal{E}$ be a refinement of $\mathcal{D}$ with the property that the first link of $\mathcal{E}$, say $E_{1}$, contains $x$ and no other link of $\mathcal{E}$ contains $x$. Let $D_{i} \in \mathcal{D}$ be the link containing $x$ and let $D_{j}$ be adjacent to $D_{i}$ in $\mathcal{D}$ such that the first link of $\mathcal{E}$ not contained in $D_{i}$ is in $D_{j}$. Call that link $E_{t}$. Let $a$ and $b$ be points of $E_{t} \cap\left(D_{j} \backslash D_{i}\right)$ that are on different composants of $K$. The existence of such points in $E_{t}$ is guaranteed by the fact that $K$ is indecomposable.

There must exist a refinement $\mathcal{F}$ of $\mathcal{E}$, with links $F_{u}$ and $F_{v}$ containing the points $a$ and $b$, respectively, such that the subchain $\left\{F_{i}\right\}_{i=u}^{v}$ meets every link of $\mathcal{E}$. This follows from the fact that $\lim _{a}\{[0,1], f\}$ is irreducible between $a$ and $b$. Also assume that the mesh of $\mathcal{F}$ is small enough to guarantee that $F_{u}$ and $F_{v}$ are contained in $D_{j} \backslash D_{i}$. Let $c$ be a positive integer such that $u<c<v$ and $F_{c} \subset E_{1}$. Clearly there must be two other positive integers $d, g$ with $u \leq d<c<g \leq v$ such that $F_{d}, F_{g} \subset E_{t} \cap\left(D_{j} \backslash D_{i}\right)$. Then $\mathcal{F}$ turns in $D_{i}$. Thus $L$ is an essential turnlink and $x$ is a folding point for $K$.

Usually inverse limits of "interesting" interval maps are indecomposable. In [5], M. Barge and J. Martin explore the relationship between complicated dynamics of $f$ and the indecomposability of $\lim _{\longleftarrow}\{[0,1], f\}$, and in the light of that paper, it is with little hesitation that we add the assumption that a certain map $f$ generates an indecomposable inverse limit space. The previous theorem demonstrates that in this quite general case, the set of folding points for the inverse limit contains all of the endpoints, and thus it contains all of the "inhomogeneities" of $\lim \{[0,1], f\}$. So $\lim _{\longleftarrow}\{[0,1], f\}$ consists of two types of points, points with solenoid-like neighborhoods and folding points. Recall, though, that the structure of Fold $(\lim \{[0,1], f\})$ is usually quite complicated. In fact it is at least as complicated as $\omega(C)$. If $\omega(C)=$ $[0,1]$, then Fold $\left(\varliminf_{\longleftarrow}\{[0,1], f\}\right)$ will be $\lim _{\longleftarrow}\{[0,1], f\}$, and if $f$ is unimodal, we

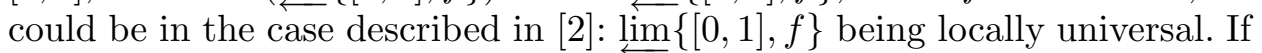
however $\omega(C)$ is a totally disconnected subset of $[0,1]$ then the set of folding points can have a quite interesting structure. We give examples of this in the next section.

7. Unimodal maps and folding points. Let $f:[0,1] \rightarrow[0,1]$ be a unimodal surjective map with critical point $c$ such that $f$ is locally eventually onto (l.e.o.). Notice that $f$ must satisfy (†). For each $x \in[0,1]$, let $I_{f}(x)$ be the itinerary of $x$ under $f$, and let $I_{f}[f(c)]=K_{f}$ denote the kneading sequence of $f$. We are primarily concerned with the structure of $\omega(c)$ in this section, and to further our examination of that set, we present the following, 
easily proved, lemmas. Some of these results have proofs in one of $[8,11,12]$, the others immediately follow from the definitions.

\section{LEMMA 7.1. If $f$ is l.e.o. then $I_{f}(x)$ is one-to-one.}

Let $\Sigma_{f}$ be the collection of possible itineraries under $f$. Then $\Sigma_{f}$ is a collection of finite and infinite sequences of symbols from the set $\{0,1, C\}$. If the sequence is finite then we have the symbol $C$ as its last term, if the sequence is infinite then every term is in $\{0,1\}$. We take $0<C<1$. The standard order to put on $\Sigma_{f}$ is the parity-lexicographical order, $\prec_{f}$. Let $s=s_{1} s_{2} \ldots$ and $t=t_{1} t_{2} \ldots$ be different itineraries in $\Sigma_{f}$ and let $n$ be the first index where they differ. If $n=1$ then $s \prec_{f} t$ if $s_{1}<t_{1}$. If $n>1$ and $s_{1} \ldots s_{n-1}$ has an even number of 1's, then define $s \prec_{f} t$ if $s_{n}<t_{n}$. If instead that string has an odd number of 1's then define $s \prec_{f} t$ if $t_{n}<s_{n}$. If $x<y$ then $I_{f}(x) \prec_{f} I_{f}(y)$ (see [11]). If $s=s_{1} s_{2} s_{3} \ldots$ is an infinite sequence of symbols and $W=s_{n} s_{n+1} \ldots s_{k}$ then we call $W$ a subword of $s$. If $W=s_{1} \ldots s_{j}$ is the first $j$ symbols of $s$ then we call $W$ an initial segment of $s$ and we write $W \leq s$. By $W^{n}$ we mean the word $W$ repeated $n$ times, and if $A$ is a word we write $W A$ for $W$ concatenated with $A$.

The shift map $\sigma$ on the set of itineraries is defined as $\sigma\left[s_{1} s_{2} \ldots\right]=$ $s_{2} s_{3} \ldots$ A sequence $s$ is shift-maximal if $\sigma^{n}(s) \prec_{f} s$ for all $n$. Since $f(c)$ is the maximum value of the unimodal function $f, K_{f}$ is shift-maximal.

An admissible sequence is an infinite sequence of 0 's and 1's or a finite sequence of 0's and 1's ending in $C$. Define $\overline{1}=0$ and $\overline{0}=1$. Then for admissible sequences $A=A^{\prime} C$ and $B=b_{0} b_{1} \ldots$, we define $A * B$ as follows:

(1) If $A$ contains an even (resp. odd) number of 1 's and $B$ is infinite, then $A * B=A^{\prime} b_{0} A^{\prime} b_{1} \ldots$ (resp. $\left.A^{\prime} \bar{b}_{0} A^{\prime} \bar{b}_{1} \ldots\right)$;

(2) If $A$ contains an even (resp. odd) number of 1 's and $B$ is finite of length $n$, then

$$
A * B=A^{\prime} b_{0} A^{\prime} b_{1} \ldots A^{\prime} b_{n-1} A^{\prime} C \quad\left(\operatorname{resp} . A^{\prime} \bar{b}_{0} A^{\prime} \bar{b}_{1} \ldots A^{\prime} \bar{b}_{n-1} A^{\prime} C\right) .
$$

If $C \prec_{f} B$ then $A \prec_{f} A * B$. Call a sequence $s=A * B$ renormalizable if $A$ and $B$ are non-empty sequences. A sequence that is not renormalizable is primary.

LEMMA 7.2 ([11, Lemma 3.1.6]). Let $(101)^{\infty} \prec_{f} W$ be an infinite sequence of 0 's and 1 's that is strictly shift-maximal and primary. Then there is a tent map $T_{a}$ with kneading sequence $W$ and $a>\sqrt{2}$.

Let $f_{a}$ denote the core of the tent map $T_{a}$, i.e. $f_{a}$ is the restriction of $T_{\alpha}$ to the interval $\left[T_{a}^{2}(c), T_{a}(c)\right]$ rescaled to be a map of $[0,1]$. Then, assuming $a \in(\sqrt{2}, 2], f_{a}$ is a l.e.o. surjection, and $\lim _{\longleftarrow}\left\{[0,1], f_{a}\right\}$ is indecomposable. All of the examples from this section will fall into this case. So the structure of Fold ${ }^{\alpha}\left(\lim _{\longleftarrow}\left\{[0,1], f_{a}\right\}\right)$ will be closely related to the structure of $\omega^{\alpha}(c)$. 
In order to analyze this set, we need a few lemmas relating the kneading sequence with the $\omega$-limit set. If $K_{f_{a}}$ is finite or eventually periodic then $\omega(c)$ is a finite set, either the periodic postcritical orbit or the periodic orbit into which $c$ is mapped. In the first instance Fold $\left(\lim ^{\longleftarrow}\left\{[0,1], f_{a}\right\}\right)$ is exactly $\operatorname{End}\left(\lim _{\longleftarrow}\{[0,1], f\}\right)$, as was demonstrated in [7]. In the second case Fold $\left(\lim _{\longleftarrow}\{[0,1], f\}\right)$ is a finite collection of points corresponding to the periodic part of the critical point's preperiodic orbit, as was demonstrated in [10]. We will be interested in the case of an infinite postcritical orbit. Since $f_{a}$ has no stable periodic points, the assumption of an infinite postcritical orbit implies that $\omega(c)$ is infinite. Since $f_{a}$ is l.e.o. and $\omega(c)$ is $f_{a^{-}}$ invariant, $\omega(c)$ is either totally disconnected, or the entire interval. The case of $\omega(c)=[0,1]$ has every point of $\varliminf_{\lfloor}\{[0,1], f\}$ in Fold $\left(\varliminf^{i m}\{[0,1], f\}\right)$ so there are no solenoid-like neighborhoods. This was the focus of [2]. We will not consider it here. We focus instead on the case of $\omega(c)$ infinite and totally disconnected.

Lemma 7.3. Let $x \in[0,1]$. Then $x \in \omega(c)$ if, and only if, for each initial segment $W$ of $I_{f}(x)$, there is an increasing sequence $\left\{n_{i}\right\}_{i \in \mathbb{N}}$ of integers such that $W \leq \sigma^{n_{i}}\left(K_{f}\right)$ for all $i \in \mathbb{N}$.

Using this lemma and the test of admissible sequences as kneading sequences (Lemma 7.2), we can proceed to construct examples. First, though, consider the following theorem due to Barge and Martin [7], and an easily proven lemma.

Theorem $7.4([7])$. End $(\underset{\lim }{\longleftarrow}\{[0,1], f\}) \neq \emptyset$ if , and only if , $c$ is recurrent under $f$.

LEMMA 7.5. If $c$ is recurrent but $\operatorname{orb}(c)$ is neither finite nor dense, then $\omega(c)$ is a Cantor set. Moreover, if $c$ is not recurrent but $\omega(c)$ contains a recurrent non-periodic point then $\omega(c)$ contains a Cantor set.

For any tent map core $f$ with $c$ recurrent but $\operatorname{orb}(c)$ not dense, the set $\operatorname{Fold}^{\alpha}(\lim \{[0,1], f\})$ is uncountable for uncountably many ordinals $\alpha$, and also $\operatorname{End}(\lim \{[0,1], f\})$ is uncountable by [9]. If instead $c$ is not recurrent but $\omega(c)$ contains a recurrent point then again $\operatorname{Fold}^{\alpha}\left(\lim _{c}\{[0,1], f\}\right)$ is uncountable for uncountably many ordinals $\alpha$, but since $c$ is not recurrent, $\operatorname{End}\left(\lim _{\lfloor}\{[0,1], f\}\right)=\emptyset$ by $[7]$.

Similarly, it is quite easy to construct a tent map core $f$ such that $\operatorname{Fold}^{0}(\underset{l i m}{\longleftarrow}\{[0,1], f\}) \neq \emptyset$ : any tent map core with a periodic critical point will suffice. It is likewise simple to construct $f$ with Fold $^{0}\left(\lim _{\longleftarrow}\{[0,1], f\}\right) \neq \emptyset$ but $\operatorname{End}\left(\lim _{\longleftarrow}\{[0,1], f\}\right)=\emptyset$ : any tent map core with a preperiodic critical point will suffice. In both of these cases though, Fold ${ }^{1}\left(\varliminf_{\longleftarrow}\{[0,1], f\}\right)=\emptyset$.

We will construct examples that fall between these two extremes. Our examples will have a finite number of levels of folding points that are count- 
ably infinite and a "top" level $\gamma$ of folding points that is finite. Notice that any time this occurs, the top level is necessarily a union of periodic orbits under every autohomeomorphism of the space. Thus points in this top level of the folding points of the inverse limit must form a finite union of periodic orbits under the shift homeomorphism. This implies that there are "special" periodic orbits under the bonding map that generate the most complicated neighborhoods of $\varliminf_{\longleftarrow}\{[0,1], f\}$.

We will now only consider the tent map cores $f_{a}$. We denote the kneading sequence of such a map by simply $K_{a}$, and if $x \in[0,1]$ the itinerary of $x$ under $f_{a}$ will be denoted by $I_{a}(x)$ rather than $I_{f_{a}}(x)$. We use $\prec$ to stand for $\prec_{f}$ where no confusion will arise.

EXAMPLE 1. Let $A=1001$ and $B=101$. Let $\left\{n_{i}\right\}_{i=1}^{\infty}$ be an increasing sequence of positive integers with $n_{1}>4$. Consider $s=A A B^{n_{1}} A B^{n_{2}} A \ldots$.

Claim 1. $(101)^{\infty} \prec s$ and $s$ is strictly shift-maximal.

Proof. Clearly $101 W \prec s$ for any word $W$ of symbols from $\{0,1\}$. Let $n \in \mathbb{N}$ and consider $\sigma^{n}(s)$. If $\sigma^{n}(s)$ begins with 0 then $\sigma^{n}(s) \prec s$. So assume it begins with 11 . Clearly $11 W \prec 10 M$ for any words $M$ and $W$. So we consider the case of $\sigma^{n}(s)$ beginning with 100. Since 1000 is not a subword of $s, \sigma^{n}(s)$ must begin with 1001. This is the word $A$. If $n$ is non-zero and $\sigma^{n}(s)$ begins with $A$ it must be followed by $B$. Clearly $\sigma^{n}(s)=A B \ldots \prec A A \ldots=s$ for this case. Thus $s$ is strictly shift-maximal.

Claim 2. $s$ is primary.

Proof. Suppose that $s=T * B$ where $T=t_{1} \ldots t_{r}$ is a finite word and $B=b_{1} b_{2} \ldots$ is an infinite word from $\{0,1\}$. Then $s=T b_{1} T b_{2} \ldots=$ $A A B^{n_{1}} A B^{n_{2}} \ldots$. Then either $T \leq A$ or $A \leq T$. Suppose that $A \leq T$. Let $j$ be large enough so that $n_{j}>\overline{2}|T|$. Then $B^{n_{j}}$ contains $T$ as a subword. This is a contradiction because $A$ is not a subword of $B^{n_{j}}$. So suppose that $T \leq A$. Then either $T=10$ or $T=100$. The first case cannot happen because $s=10011001 \ldots \neq T u_{1} T \ldots=10 u_{1} 10$ regardless of our choice for $u_{1}$. If $T=100$ then we find that eventually 100 is a subword of $101^{n_{j}}$, which is a contradiction. Thus $s$ is primary.

Claim 3. There is a parameter $z \in(\sqrt{2}, 2]$ such that $K\left(f_{z}\right)=s$.

Proof. $s$ is primary and shift-maximal and $(101)^{\infty} \prec s$. Thus by Lemma 7.2 such a parameter exists.

Now we will analyze the structure of $\omega(c)$ for the core of this tent map. We do this by appealing to Lemma 7.3, and finding points $x$ with itineraries such that every subword of $I_{z}(x)$ occurs infinitely often in $K_{z}$.

Let $\alpha, \beta, \gamma \in[0,1]$ be such that $I_{z}(\alpha)=(101)^{\infty}, I_{z}(\beta)=(011)^{\infty}$ and $I_{z}(\gamma)=(110)^{\infty}$. Clearly these are the points of a period three orbit. We will 
show that $\omega^{1}(c)=\{\alpha, \beta, \gamma\}$. For each $k \in \mathbb{N} \cup\{0\}$ define $a_{k}, b_{k}, c_{k} \in[0,1]$ by

$$
\begin{aligned}
I_{z}\left(a_{k}\right) & =(101)^{k} A(101)^{\infty}, \\
I_{z}\left(b_{k}\right) & =\sigma\left[(101)^{k}\right] A(101)^{\infty}, \\
I_{z}\left(c_{k}\right) & =\sigma^{2}\left[(101)^{k}\right] A(101)^{\infty} .
\end{aligned}
$$

For $0 \leq j \leq 4$, define $d_{j} \in[0,1]$ by

$$
I_{z}\left(d_{j}\right)=\sigma^{j}(A)(101)^{\infty} .
$$

Claim 4. $\omega(c)=\{\alpha, \beta, \gamma\} \cup\left\{a_{k}, b_{k}, c_{k}\right\}_{k=0}^{\infty} \cup\left\{d_{j}\right\}_{j=0}^{4}$.

Proof. Set $Q=\{\alpha, \beta, \gamma\} \cup\left\{a_{k}, b_{k}, c_{k}\right\}_{k=0}^{\infty} \cup\left\{d_{j}\right\}_{j=0}^{4}$. Clearly, by Lemma 7.3, $Q \subseteq \omega(c)$. Suppose that $w \in \omega(c)$ and $I_{z}(w)=r_{1} r_{2} r_{3} \ldots$ If $I_{z}(w)$ does not contain $A$ and does not begin with $\sigma(A)$, then clearly $I_{z}(w)=$ $(101)^{\infty},(011)^{\infty}$ or $(110)^{\infty}$.

Suppose that $I_{z}(w)$ contains $A$. Notice that for $m=0,1$, the word $\sigma^{m}(A) A$ cannot be a subword of $I_{z}(w)$, because that word only occurs once in $K_{z}$. So now suppose that $I_{z}(w)$ contains $A$ twice, but separated by some word $V$, i.e. $A V A$ is a subword of $I_{z}(w)$. Let $j$ be large enough so that $n_{j}>2|V|$. Then after the first occurrence of $B^{n_{j}}$ there must be an occurrence of $A V A$. But all of the words past $B^{n_{j}}$ in $K_{z}$ containing two copies of $A$ are of the form $A B^{n_{k}} A$, where $k>j$. Clearly none of these equal $A V A$. Thus $I_{z}(W)$ can contain at most one occurrence of $A$.

In a previous paragraph we discussed the possibility of no occurrence of $A$. Now suppose that $I_{z}(w)$ contains $A$ as a subword. Assume that $I_{z}(w)=$ $r_{1} r_{2} \ldots r_{n} A r_{n+5} \ldots$ Clearly, this must be $r_{1} \ldots r_{n} A(101)^{\infty}$, because if any other string occurs after $A$ then we would have an easy contradiction. Now consider the possibilities for the symbols before $A$. It is not hard to see that these must be of the form $(101)^{k}, \sigma\left[(101)^{k}\right]$ or $\sigma^{2}\left[(101)^{k}\right]$ for $k \in \mathbb{N} \cup\{0\}$. Thus $Q=\omega(c)$.

Claim 5. $\{\alpha, \beta, \gamma\}=\omega^{\prime}(c)$.

Proof. Let $w \in[0,1]$. For each word $W$ of finite length from $I_{z}(w)$ there is a subinterval $S_{W}$ of $[0,1]$ such that $x \in S_{W}$ if, and only if, $I_{z}(x)=W V$ for some word $V$. The diameter of $S_{W}$ decreases to zero as the length of $W$ increases to infinity. Thus it is not hard to see that $a_{k} \rightarrow \alpha, b_{k} \rightarrow \beta$ and $c_{k} \rightarrow \gamma$ as $k \rightarrow \infty$. The claim follows.

In this case $f_{z}$ is well-founded. To see this let $x_{0} \in \omega(c)$. Call an infinite sequence $\left(x_{0}, x_{-1}, x_{-2}, \ldots\right)$ permitted provided $f\left(x_{-i}\right)=x_{-(i-1)}$ and $x_{-i} \in \omega(c)$ for each $i \in \mathbb{N}$. Notice that the collection of permitted sequences is the collection of points in $\lim _{\longleftarrow}\left\{[0,1], f_{z}\right\}$ that always project into $\omega(c)$. Hence by previous results this collection is the collection of folding points for $\lim _{\longleftarrow}\left\{[0,1], f_{z}\right\}$. Assume $n \in \mathbb{N}$ and $x_{0} \in \omega^{n}(c)$. Call a permitted sequence 
$\left(x_{0}, x_{-1}, x_{-2}, \ldots\right) n$-permitted provided that $x_{-i} \in \omega^{n}(c)$ for all $i \in \mathbb{N}$. If $n=1$ and $x_{0} \in \omega^{1}(c)$ then by the structure of $\omega(c)$ it is easy to see that there is exactly one 1-permitted sequence starting with $x_{0}$. However there are countably many permitted sequences for $x_{0}$ and there are three possible $x_{0} \in \omega^{1}(c)$ to choose from. Thus there are three points in $\lim _{\longleftarrow}\left\{[0,1], f_{z}\right\}$ which always project into $\omega^{1}(c)$. For any other permitted sequence starting with $x_{0}$ there is a least integer $j$ such that $x_{-j} \in \omega^{0}(c)$ and $\left(x_{-j}, x_{-j-1}, \ldots\right)$ is a 0 -permitted sequence. Let $\widehat{\alpha}, \widehat{\beta}, \widehat{\gamma} \in \lim \{[0,1], f\}$ be the points that correspond to the 1-permitted sequence starting with $\alpha, \beta$, or $\gamma$ respectively. Given $\alpha$ and a positive integer $j$ it is not hard to see that there is exactly one permitted sequence starting with $\alpha$ such that $j$ is the least integer with $\left(x_{-j}, x_{-j-1}, \ldots\right)$ a 0 -permitted sequence. Let $\widehat{a}_{j}$ be the point in the inverse limit that corresponds to this permitted sequence. Clearly $\widehat{a}_{j}$ is isolated and $\widehat{a}_{j} \rightarrow \widehat{\alpha}$ as $j \rightarrow \infty$. Similar sequences of points can be constructed for both $\widehat{\beta}$ and $\widehat{\gamma}$. Thus Fold ${ }^{0}\left(\varliminf_{\longleftarrow}\left\{[0,1], f_{z}\right\}\right)$ is countably infinite and Fold ${ }^{1}\left(\lim _{\longleftarrow}\left\{[0,1], f_{z}\right\}\right)$ contains three points, $\widehat{\alpha}, \widehat{\beta}$ and $\widehat{\gamma}$. Therefore, $f_{z}$ is well-founded.

Clearly there was nothing special about our choice of $A$ or $B$, and it is apparent that $B$ is the portion of the kneading sequence that generates the points in $\operatorname{Fold}^{1}\left(\lim \left\{[0,1], f_{z}\right\}\right)$. We could have made $B$ a longer periodic itinerary and then Fold $^{1}\left(\lim \left\{[0,1], f_{z}\right\}\right)$ would have had more points. In order to maintain $s$ being shift-maximal and primary we would have had to lengthen $A$ accordingly. We could also have chosen $n$ different periodic itineraries $B_{1}, \ldots, B_{n}$ and a word $A$ so that Fold ${ }^{1}\left(\lim _{\longleftarrow}\left\{[0,1], f_{z}\right\}\right)$ would correspond to the orbits of $n$ different periodic points.

In order to construct a map that generates non-trivial Fold ${ }^{2}$ points we must alter the above construction in a significant way. We will present that construction. The interested reader can easily iterate our technique to generate a well-founded tent map core $f$ with Fold $^{n}\left(\lim _{\longleftarrow}\{[0,1], f\}\right)$ a finite set corresponding to finitely many periodic orbits for any positive integer $n$.

ExAmple 2. Let $A=1001, B=101,\left\{m_{i}\right\}_{i=1}^{\infty}$ be an increasing sequence of positive integers, and let $C_{i}=A B^{i} A$. Let $t$ be the sequence of positive integers given by

$$
t=t_{1}, t_{2}, t_{3}, \ldots=1,1,2,1,2,3,1,2,3,4,1,2,3,4,5,1,2,3,4,5,6, \ldots .
$$

Define $s$ by

$$
s=A C_{t_{1}} B^{m_{1}} C_{t_{2}} B^{m_{2}} C_{t_{3}} B^{m_{3}} C_{t_{4}} \ldots
$$

It is not hard to see that $s$ is strictly shift-maximal and primary. Thus there is a parameter $y \in(\sqrt{2}, 2]$ such that $K_{y}=s$. Let now $c$ be the critical point of the tent map core $f_{y}$. Consider $\omega(c)$. 
Define $\alpha, \beta, \gamma \in[0,1]$ by $I_{y}(\alpha)=(101)^{\infty}, I_{y}(\beta)=(011)^{\infty}$ and $I_{y}(\gamma)=$ $(110)^{\infty}$. We will show that $\{\alpha, \beta, \gamma\}=\omega^{2}(c)$ and thus they correspond to the three points in $\operatorname{Fold}^{2}\left(\lim _{\longleftarrow}\left\{[0,1], f_{y}\right\}\right)$.

As before, given $k \in \mathbb{N} \cup\{0\}$, let $a_{k}, b_{k}, c_{k} \in[0,1]$ be given by

$$
\begin{aligned}
& I_{y}\left(a_{k}\right)=(101)^{k} A(101)^{\infty}, \\
& I_{y}\left(b_{k}\right)=\sigma\left[(101)^{k}\right] A(101)^{\infty}, \\
& I_{y}\left(c_{k}\right)=\sigma^{2}\left[(101)^{k}\right] A(101)^{\infty} .
\end{aligned}
$$

For $0 \leq j \leq 4$, define $d_{j} \in[0,1]$ by

$$
I_{y}\left(d_{j}\right)=\sigma^{j}(A)(101)^{\infty} .
$$

For the same reason as previously all of these points will be in $\omega(c)$ and still $a_{k} \rightarrow \alpha, b_{k} \rightarrow \beta$ and $c_{k} \rightarrow \gamma$. However, now we have $\left\{a_{k}, b_{k}, c_{k}\right\}_{k=1}^{\infty}=\omega^{\prime}(c)$. To see this, let $k \in \mathbb{N} \cup\{0\}$ and let $n \in \mathbb{N}$. Notice that $C_{n}=A B^{n} A$ occurs infinitely often in $s$, and in fact $B^{k} C_{n}=B^{k} A B^{n} A, \sigma\left(B^{k}\right) C_{n}=$ $\sigma\left(B^{k}\right) A B^{n} A$, and $\sigma^{2}\left(B^{k}\right) C_{n}=\sigma^{2}\left(B^{k}\right) A B^{n} A$ occurs infinitely often in $s$. So define $a_{n}^{k}, b_{n}^{k}, c_{n}^{k} \in[0,1]$ by

$$
\begin{aligned}
I_{y}\left(a_{n}^{k}\right) & =(101)^{k} C_{n}(101)^{\infty}=(101)^{k} A(101)^{n} A(101)^{\infty}, \\
I_{y}\left(b_{n}^{k}\right) & =\sigma\left[(101)^{k}\right] C_{n}(101)^{\infty}=\sigma\left[(101)^{k}\right] A(101)^{n} A(101)^{\infty}, \\
I_{y}\left(c_{n}^{k}\right) & =\sigma^{2}\left[(101)^{k}\right] C_{n}(101)^{\infty}=\sigma^{2}\left[(101)^{k}\right] A(101)^{n} A(101)^{\infty} .
\end{aligned}
$$

It is not hard to see that $\left\{a_{n}^{k}, b_{n}^{k}, c_{n}^{k}\right\}_{k=0, n=1}^{\infty} \subseteq \omega(c)$, and $a_{n}^{k} \rightarrow a_{k}, b_{n}^{k} \rightarrow b_{k}$ and $c_{n}^{k} \rightarrow c_{k}$ as $n \rightarrow \infty$. Thus $\left\{a_{k}, b_{k}, c_{k}\right\}_{k=0}^{\infty} \subseteq \omega^{\prime}(c)$. It can be shown that the only points left in $\omega(c)$ form a finite collection of isolated points. Thus $\omega^{\prime}(c)=\left\{a_{k}, b_{k}, c_{k}\right\}_{k=0}^{\infty}$ and $\omega^{2}(c)=\{\alpha, \beta, \gamma\}$. It can also be shown in the same manner as before that $f_{y}$ is well-founded. Hence $f_{y}$ is the core of a tent map with Fold $\left(\varliminf_{\mathfrak{m}}\left\{[0,1], f_{y}\right\}\right)$ a countably infinite set, $\operatorname{Fold}^{1}\left(\lim _{\longleftarrow}\left\{[0,1], f_{y}\right\}\right)$ a countably infinite set and Fold ${ }^{2}\left(\varliminf_{\longleftarrow}\left\{[0,1], f_{y}\right\}\right)$ three points, each of which project into $\{\alpha, \beta, \gamma\}$.

It is easy to see that we could have chosen a different word $B$ that could have led to more points in $\operatorname{Fold}^{2}\left(\lim _{\longleftarrow}\left\{[0,1], f_{y}\right\}\right)$, and we could have even chosen finitely many periodic itineraries $B_{1}, \ldots, B_{n}$ that would have led to a slightly more rich structure for $\operatorname{Fold}^{2}\left(\varliminf_{\longleftarrow}\left\{[0,1], f_{y}\right\}\right)$. Of course in each case, $A$ would have to be altered slightly.

For more examples of such structures in $\omega(c)$ see [17, 18]. We will end the paper with a brief description of how to alter the previous example into tent maps that generate inverse limit spaces with a non-empty but finite collection of $n$-folding points for any positive integer $n$.

Example 3. Given $A, B,\left\{m_{i}\right\}_{i \in \mathbb{N}}$ and $t_{i}$ defined as in Example 2, let $N=\left\{\left(\alpha_{1}, \ldots, \alpha_{j}\right): \alpha_{i} \in \mathbb{N}\right.$ and $\left.j \leq n\right\}$. Clearly $N$ is countable, so enumer- 
ate $N$ by $N=\left\{\alpha_{1}, \alpha_{2}, \ldots\right\}$, where $\alpha_{i}=\left(\alpha_{1}^{i}, \ldots, \alpha_{k_{i}}^{i}\right)$. Let

$$
C_{i}=A B^{\alpha_{1}^{i}} A B^{\alpha_{2}^{i}} A \ldots A B^{\alpha_{k_{i}}^{i}} A .
$$

Define

$$
s=A A C_{t_{1}} B^{m_{1}} C_{t_{2}} B^{m_{2}} C_{t_{3}} B^{m_{4}} \ldots
$$

Using techniques from previous examples it can be shown that $s$ is strictly shift-maximal and primary. Thus there is a parameter $v \in(\sqrt{2}, 2]$ such that $K_{v}=s$. Let $c$ be the critical point for $f_{v}$. It can be shown that $\omega^{n}(c)=$ $\{\alpha, \beta, \gamma\}$, where $I_{v}(\alpha)=B^{\infty}, I_{v}(\beta)=\sigma(B)^{\infty}$ and $I_{v}(\gamma)=\sigma^{2}(B)^{\infty}$, and that $f_{v}$ is well-founded.

In an upcoming paper we completely classify all possible topological structures of these $\omega$-limit sets, giving examples of $\alpha$-folding points for countable transfinite ordinals $\alpha$. We also give examples in which $\mid$ Fold $\left.\left(\lim _{\longleftarrow}\left\{[0,1], f_{a}\right\}\right)\right|_{\mathrm{CB}}$ $=\alpha$ is a transfinite ordinal, but $\operatorname{Fold}^{\alpha}\left(\lim _{\longleftarrow}^{\log }\left\{[0,1], f_{a}\right\}\right)$ is a Cantor set rather than a finite collection of periodic orbits.

\section{References}

[1] J. E. Anderson and I. F. Putnam, Topological invariants for substitution tilings and their associated $C^{*}$-algebras, Ergodic Theory Dynam. Systems 18 (1998), 509-537.

[2] M. Barge, K. Brucks and B. Diamond, Self-similarity in inverse limit spaces of the tent family, Proc. Amer. Math. Soc. 124 (1996), 3563-3570.

[3] M. Barge and B. Diamond, A complete invariant for the topology of one-dimensional substitution tiling spaces, Ergodic Theory Dynam. Systems 21 (2001), 1333-1358.

[4] M. Barge and S. Holte, Nearly one-dimensional Hénon attractors and inverse limits, Nonlinearity 8 (1995), 29-42.

[5] M. Barge and J. Martin, Chaos, periodicity, and snakelike continua, Trans. Amer. Math. Soc. 289 (1985), 355-365.

[6] —, - The construction of global attractors, Proc. Amer. Math. Soc. 110 (1990), $523-525$.

[7] - - - Endpoints of inverse limit spaces and dynamics, in: Continua (Cincinnati, OH, 1994), Dekker, New York, 1995, 165-182.

[8] L. S. Block and W. A. Coppel, Dynamics in One Dimension, Springer, Berlin, 1992.

[9] H. Bruin, Planar embeddings of inverse limit spaces of unimodal maps, Topology Appl. 96 (1999), 191-208.

[10] - Inverse limit spaces of post-critically finite tent maps, Fund. Math. 165 (2000), $125-138$.

[11] P. Collet and J.-P. Eckmann, Iterated Maps on the Interval as Dynamical Systems, Birkhäuser Boston, 1980.

[12] W. de Melo and S. van Strien, One-Dimensional Dynamics, Ergeb. Math. Grenzgeb. 25, Springer, Berlin, 1993.

[13] J. G. Hocking and G. S. Young, Topology, 2nd ed., Dover Publ., New York, 1988.

[14] W. T. Ingram, An atriodic tree-like continuum with positive span, Fund. Math. 77 (1972), 99-107.

[15] —, Inverse Limits, Soc. Mat. Mexicana, México, 2000. 
[16] A. S. Kechris, Classical Descriptive Set Theory, Grad. Texts in Math. 156, Springer, New York, 1995.

[17] B. Raines, One-dimensional dynamics and inverse limits, D.Phil. thesis, Oxford Univ., 2002.

[18] - Inverse limits of tent maps without the pseudo-orbit shadowing property, Topology Proc. 27 (2003), 1-9.

[19] J. van Mill and G. M. Reed (eds.), Open Problems in Topology, North-Holland, Amsterdam, 1990.

[20] R. F. Williams, One-dimensional non-wandering sets, Topology 6 (1967), 473-487.

[21] - Classification of one dimensional attractors, in: Global Analysis (Berkeley, CA, 1968), Proc. Sympos. Pure Math. 14, Amer. Math. Soc., Providence, RI, 1970, 341-361

[22] - The "DA" maps of Smale and structural stability, in: Global Analysis (Berkeley, CA, 1968), Proc. Sympos. Pure Math. 14, Amer. Math. Soc., Providence, RI, 1970, 329-334.

[23] I. Yi, Canonical symbolic dynamics for one-dimensional generalized solenoids, Trans. Amer. Math. Soc. 353 (2001), 3741-3767.

Mathematical Institute

University of Oxford

Oxford OX1 3LB, UK
Current address:

Department of Mathematics

Baylor University

Waco, TX 76798-7328, U.S.A.

E-mail: Brian_Raines@baylor.edu

Received 22 October 2003;

in revised form 21 July 2004 